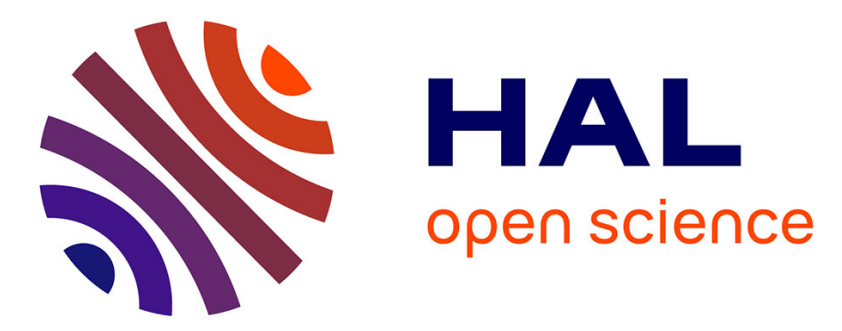

\title{
On the use of SEM correlative tools for in situ mechanical tests
}

\author{
Qiwei Shi, Stéphane Roux, Félix Latourte, François Hild, Dominique \\ Loisnard, Nicolas Brynaert
}

\section{- To cite this version:}

Qiwei Shi, Stéphane Roux, Félix Latourte, François Hild, Dominique Loisnard, et al.. On the use of SEM correlative tools for in situ mechanical tests. Ultramicroscopy, 2018, 184, Part A, pp.71-87. 10.1016/j.ultramic.2017.08.005 . hal-01674581

\section{HAL Id: hal-01674581 \\ https://hal.science/hal-01674581}

Submitted on 23 Apr 2018

HAL is a multi-disciplinary open access archive for the deposit and dissemination of scientific research documents, whether they are published or not. The documents may come from teaching and research institutions in France or abroad, or from public or private research centers.
L'archive ouverte pluridisciplinaire HAL, est destinée au dépôt et à la diffusion de documents scientifiques de niveau recherche, publiés ou non, émanant des établissements d'enseignement et de recherche français ou étrangers, des laboratoires publics ou privés. 


\title{
On the use of SEM correlative tools for in situ mechanical tests
}

\author{
Qiwei SHI $^{\mathrm{a}, \mathrm{b}, *}$, Stéphane Roux ${ }^{\mathrm{a}}$, Félix Latourte ${ }^{\mathrm{b}}$, François Hild ${ }^{\mathrm{a}}$, Dominique Loisnard ${ }^{\mathrm{b}}$, Nicolas Brynaert $^{\mathrm{b}}$ \\ ${ }^{a} L M T$, ENS Paris-Saclay / CNRS / Université Paris-Saclay, \\ 61 avenue du Président Wilson, F-94235 Cachan (FRANCE) \\ ${ }^{b}$ EDF R\&D, Site des Renardières, \\ avenue des Renardières, Ecuelles, F-77818 Moret-sur-Loing (FRANCE)
}

\begin{abstract}
In situ SEM mechanical tests are key to study crystal plasticity. In particular, imaging and diffraction (EBSD) allow microstructure and surface kinematics to be monitored all along the test. However, to get a full benefit from different modalities, it is necessary to register all images and crystallographic orientation maps from EBSD into the same frame. Different correlative approaches tracking either Pt surface markings, crystal orientations or grain boundaries, allow such registrations to be performed and displacement as well as rotation fields to be measured, a primary information for crystal plasticity identification. However, the different contrasts that are captured in different modalities and unavoidable stage motions also give rise to artifacts that are to be corrected to register the different information onto the same material points. The same image correlation tools reveal very powerful to correct such artifacts. Illustrated by an in situ uniaxial tensile test performed on a bainitic-ferritic steel sample, recent advances in image correlation techniques are reviewed and shown to provide a comprehensive picture of local strain and rotation maps.
\end{abstract}

Keywords: BSE, EBSD, Crystal plasticity, Digital image correlation, in situ tension, SEM

\section{Introduction}

Scanning Electron Microscopy allows contrasted images to be acquired with sub-micrometer spatial resolution at the surface of metals, using Secondary Electron (SE) imaging mode, mostly sensitive to topography. Back-Scattered Electron (BSE) imaging is sensitive to topography or chemical contrast depending on the detector configuration. 5 The latter case is the most favorable for kinematic measurements since brightness changes due to out-of plane displacements are essentially prevented. New generations of Scanning Electron Microscopes (SEM) with field emission guns exhibit far less imaging distortions than their predecessors based on Tungsten probe technology [1, and are therefore very well adapted to performing digital image correlation (DIC) measurements [2, 3, 4.

Another imaging mode providing key information when studying crystal deformation is Electron Back-Scattered

10 Diffraction (EBSD). Sensitive to crystal orientation, EBSD allows rotation fields to be tracked at the surface of a polycrystalline specimen subjected to straining [5. Measuring rotation and more specifically its gradients is important to address plasticity problems at the grain scale, since rotation gradients are induced by geometrically necessary dislocations involved in strain gradient plasticity models [6, 7].

In situ mechanical tests often rely on the combined use of BSE and EBSD images, but these two images are acquired with two different specimen configurations. The surface is normal to the electron beam for BSE images and is subsequently tilted for EBSD imaging. Matching the coordinates system of the two images is an issue seldom reported in the literature. A commonly adopted procedure is to use indent marks, which are visible in the two images, to find the transformation between the images using simple shape functions (e.g., rotations, magnification changes/dilations [ 8 ).

Kinematic measurement by DIC requires gray level images ideally with high local contrast. Apart from natural contrast of the sample surface (e.g., surface roughness), different marking techniques are available to produce a fitting artificial contrast for SEM studies, such as the deposition of microgrids by microlithography [9], random patterns generated by nanoparticle deposition techniques [10] or UV-photolithography [11. To the best knowledge of the authors, all the artificial patterns serve as contrasts in SE/BSE images, and no report exists concerning the

\footnotetext{
${ }^{*}$ Corresponding author

Email address: qshi@lmt.ens-cachan.fr (Qiwei SHI)
} 
exploitation of its contrast in EBSD images. Besides, the mechanism of trace generation in EBSD images has not been investigated.

An alternative solution is to deposit in situ computer-generated patterns onto the specimen surface by microlithography with a precise knowledge of the reference pattern 3. Another advantage of the in situ deposition is that the size, shape and thickness of the speckles are easily and precisely controlled by the SEM. As information 30 depth is bigger in EBSD than SE/BSE images, and the spatial resolution of EBSD is not as good as SE/BSE images, larger and thicker speckles are necessary to create sufficient contrast in EBSD images for DIC purposes. This paper presents a work to generate a visible random pattern in both SE/BSE and EBSD images and employs multiple correlative tools to track the pattern in these imaging modes during the in situ test, thereby generating very rich experimental information about the sample.

The characterization of SEM errors is critical when images are quantitatively analyzed. Imaging distortion of SEM has been characterized by DIC 3 on samples with in situ deposited speckles. Slow scan direction error in atomic force microscpy (AFM) has been quantified and corrected [12, 13, by capturing two images with orthogonal scan directions. A hypothesis has been made that the information acquired for each scanned line is reliable. EBSD acquisition uncertainty, both spatial distortion and orientation resolution, should be analyzed too. A detailed orientation uncertainty analysis 14 estimates the angular resolution at $0.5^{\circ}$, which has been confirmed by experimental measurements 15 .

For tilted samples, distortion of EBSD images has been pointed out [16, 17, and a geometric transformation has been proposed to correct the distortion based on SEM images on non tilted samples. The tilt correction has since been incorporated as a basic function in standard SEMs. Trapezoidal distortion has been analyzed theoretically [18, 19]. However, the uncertainty in tilt correction has not been studied.

The present study aims at coupling crystal deformation and rotation measurements of a tensile test performed in situ (i.e., in an SEM). Surface deformations are measured thanks to DIC [9, 20, 8, with a specific implementation already proven well adapted to polycrystals 21. More recently, it was shown that crystal rotation fields can also be measured on surfaces by registering EBSD images via so-called quaternion correlation 5 . EBSD images have been overlaid manually on DIC strain map to study the relation between the texture and fatigue properties 22 . Accurate matching of the two kinds of images is necessary to describe the microstructure of polycrystals and more importantly, its changes during mechanical loadings. Rotations measured by EBSD and strains evaluated by DIC were compared 23] for an austenitic steel with large grain size. Matching EBSD and BSE images was thus not mandatory in that work since only few grains were studied.

55 Since comparisons will be performed herein for a bainitic steel with a much finer microstructure, rotation and strain fields have to be expressed very precisely with respect to each other. The matching of BSE and EBSD images will rely on the presence of the same speckle pattern produced by lithography in the images captured with different specimen positions and detectors. Efforts will be devoted to the understanding of electronic processes leading to image capture, thereby allowing for good correlation between images acquired with different modalities, and in turn sound comparisons between plastic slip and lattice rotation. The paper is organized as follows. Section 2 details the experimental procedure. A very brief introduction to DIC is provided in Section 3 Section 4 is dedicated to the treatment of BSE images, and Section 5 to that of EBSD images. Matching EBSD and BSE images and its benefit will be discussed in Section 6 .

\section{Experimental settings}

The studied material, A508cl3 (i.e., 16MND5 in French nomenclature), which is a bainitic-ferritic low-alloyed steel used in the nuclear industry, is subjected to in situ uniaxial tension. Two flat tensile specimens with a gage zone of volume $10 \times 1.5 \times 1 \mathrm{~mm}^{3}$ are machined. For imaging purposes, mechanical polishing of the sample surface is performed with silk cloths and diamond suspension down to $0.25 \mu \mathrm{m}$ followed by finishing with $40 \mathrm{~nm}$ colloidal silica suspension for 25 minutes.

70 A random platinum speckle has been generated and deposited in situ onto the sample surface. The theoretical position of the speckles is shown in Figure 1. Note that the $x$ direction is vertical and $y$ direction horizontal, and the coordination origin is at the top left of the image. This convention will be used in all the following images of the paper. A platinum square of $110 \times 110 \mu \mathrm{m}^{2}$ encompassing the speckles has also been deposited, to facilitate the sample repositioning during the tensile test. The shape of the square also indicates intuitively the deformation, or the distortion of captured images. The diameter of the speckles is set to $300 \mathrm{~nm}$ and their thickness is $75 \mathrm{~nm}$. These parameters are optimized prior to the test, by trial and error, to make the speckles visible in image quality (IQ) maps and invisible in orientation maps associated with EBSD analyses. The speckles cover a Region of Interest of area $105 \times 105 \mu \mathrm{m}^{2}$. 


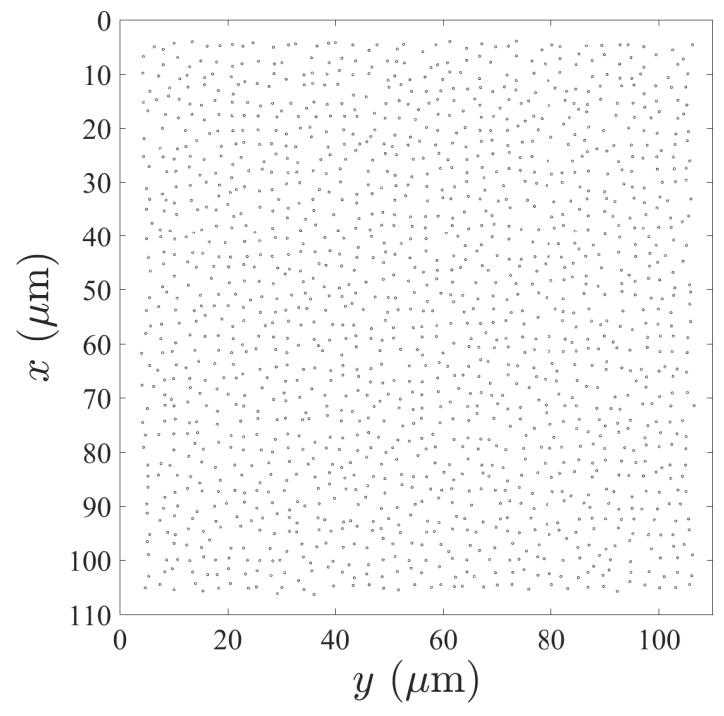

Figure 1: Theoretical positions of randomly generated speckles

The SEM used in this study is of type TESCAN Mira3 600. BSE detector in Z-contrast has been used with an acceleration voltage of $30 \mathrm{kV}$ and a working distance of $17 \mathrm{~mm}$. The physical size of one pixel is $50 \mathrm{~nm}$, which is close to the spatial resolution limit of EBSD acquisitions. This very fine resolution is chosen because 16MND5 has a very fine microstructure, with $1-2 \mu \mathrm{m}$ thick bainitic laths. The scanned area $\left(110 \times 110 \mu \mathrm{m}^{2}\right)$ has a definition of $2200 \times 2200$ pixels. Two successive EBSD acquisitions have been performed for the undeformed sample and another two acquisitions for the deformed sample. Each acquisition duration is about $21 \mathrm{~h}$, allowing enough electrons to be due to temperature changes between day and night. The data obtained by EBSD acquisitions and processed with EDAX OIM ${ }^{\mathrm{M}}$ software are used as a starting point of EBSD analyses.

An in situ tensile test has been performed on the sample in the horizontal position. The macroscopic strain rate is set to $4 \times 10^{-4} \mathrm{~s}^{-1}$ up to $6 \%$ longitudinal strain. Series of SE and BSE images have been acquired during the whole test. Scan rotation has been set successively to $0^{\circ}$ and $90^{\circ}$ for each step. The image definition is $3072 \times 3072$ pixels for a surface area of $130 \times 130 \mu \mathrm{m}^{2}$. The images are acquired with an acceleration voltage of $30 \mathrm{kV}$ and a working distance of $13 \mathrm{~mm}$. It is worth noting that EBSD acquisitions necessitate a tilted configuration (surface normal inclined with respect to the SEM column by about $70^{\circ}$ ), and are not accessible during the in situ tensile test.

Let us mention that another strategy was tested to facilitate the registration of EBSD and surface imaging. The mechanical stage was positioned so that the sample surface remained inclined at about $70^{\circ}$ (as required for EBSD acquisition) during the entire test. The tilt correction of the SEM was used to revert to in-plane coordinates. However, registration performed on a sequence of images acquired during the mechanical test showed spurious stripes on the displacement field (at subpixel amplitudes) that originated from the built-in tilt correction. Thus, although the idea was appealing, and in spite of a very careful and otherwise successful mechanical test, tilt correction precluded the use of imaging in a quantitative manner, and this option was not investigated further.

\section{Brief introduction to DIC}

For a few recent decades image-based measurement methods, known as digital image correlation, have been developed and widely used 24. A variety of DIC techniques have been proposed to register different types of images, for example 2D-DIC [25, 9, 20, 2, 1, 3D-DIC [26, 27, 28, digital volume correlation 229, 30, 31, 32, and quaternion correlation [5].

The same principal applies to all DIC techniques. The input of DIC methods is image pairs, denoted as $f(\boldsymbol{x})$ and $g(\boldsymbol{x})$, with some features, for example marking or topography, by extension grain orientation for quaternion correlation. DIC techniques search for displacement field $\boldsymbol{u}(\boldsymbol{x})$ that relates $f$ and $g$ by invoking gray level conservation

$$
f(\boldsymbol{x}) \approx g(\boldsymbol{x}+\boldsymbol{u}(\boldsymbol{x}))
$$


The outputs are i) the displacement field between the 2 input images plus possibly extra corrections such as gray levels; ii) the difference between $f(\boldsymbol{x})$ and $g(\boldsymbol{x}+\boldsymbol{u}(\boldsymbol{x}))$, or also called residuals, showing how good/bad the registration was performed. It is worth noting that in the case of quaternion correlation, the residual corresponds to the crystal disorientation between 2 images. The way the gray level conservation (1) is sought depends on the chosen displacement kinematics and over which part of the image it is applied [33.

The present paper employs intensively 2D-DIC and quaternion correlation to perform the measurements for different types of images taken during the in situ mechanical test. A regularized and global DIC algorithm is used and the DIC procedure is characterized by its spatial resolution given by the largest length scale between the displacement discretization (here an unstructured FE mesh made of 3-noded triangular elements) and a regularization length promoting small scale smoothness 34 .

\section{Analysis of SEM images}

\subsection{SEM scan orientation artifacts on horizontal sample}

According to previous studies, images acquired by scanning microscopy, e.g., electron microscopy [2, 1] and atomic force microscopy (AFM) may suffer from distortions/scan drift, especially in the slow scan direction 35 . To assess such distortions it has been proposed to capture images in different scan directions [36, and to combine them, relying on fast scan lines, to get an image corrected from such line to line artifacts [13. In this last reference, artificial strains due to such slow scan drift was observed to reach $16 \%$ for AFM images.

In the present experiment, all SEM images are taken with a scan rotation of $0^{\circ}$ and $90^{\circ}$ to correct for potential direction-related distortions. For all images with a scan rotation of $0^{\circ}$, the 'fast' scan direction is $y$ and lines at increasing $x$ positions are progressively accumulated ( $x$ is said to be the 'slow' scan direction). Conversely, for the scan rotation of $90^{\circ}$, the fast scan direction is $x$ and line data are collected at increasing $y$ coordinates. As a result, images taken with scan rotations $0^{\circ}$ and $90^{\circ}$ are comparable only after rotating an image by $90^{\circ}$. Figures 2(a) and 2(b) show the BSE images of the reference state of the sample. Figure 2(c) shows the $90^{\circ}$ acquisition (Figure 2(b) exactly rotated by an angle of $90^{\circ}$ anti-clock wise. The difference between Figures 2(a) and 2(c) is hardly detectable with bare eyes.

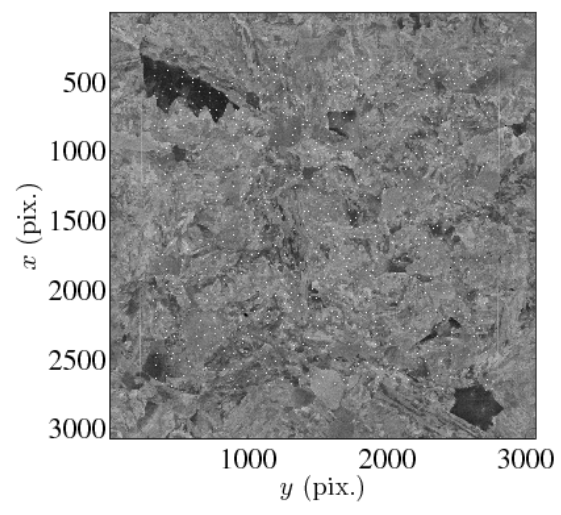

(a) $0^{\circ}$-scan image

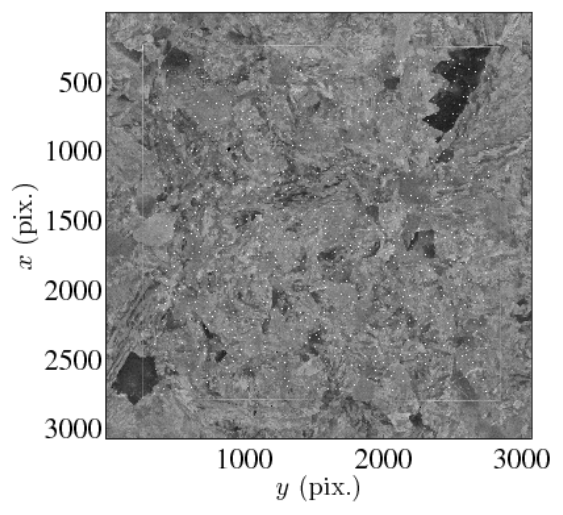

(b) $90^{\circ}$-scan image

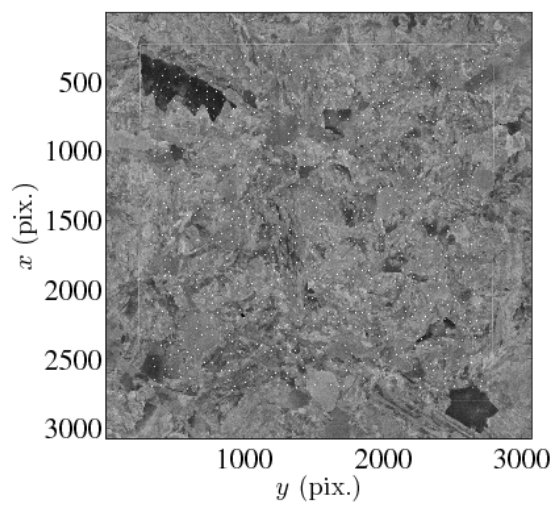

(c) Rotated $90^{\circ}$-scan image

Figure 2: BSE images of the sample in the reference configuration acquired with a scan direction of (a) $0{ }^{\circ}$ and (b) $90^{\circ}$. (c) Numerically rotated image (b) by $90^{\circ}$ anti-clockwise. The pixel size is $42 \mathrm{~nm}$ for all images

DIC has been run to register images acquired with different scan directions (Figures 2(a) and 2(c)). Triangular elements with characteristic length of 25 pixels are adopted, and the regularization length is set to 200 pixels to reduce the measurement uncertainty as the spatial resolution is not an issue herein.

An example of DIC result with the BSE images of the reference state is shown in Figure 3 . Figure 3(a) shows the raw difference between Figures 2(a) and 2(c), for which a clear difference is observed. A small region in Figure 3(a) is free of speckles, while in its neighborhood speckles gradually appear. This phenomenon implies a slight yet persistent distortion between the two studied images. Figure 3(b) shows the gray level residual field at convergence of the DIC routine. Although some patterns can still be seen, the level of residuals has been greatly decreased from the initial difference. The histogram is shown in Figure 3(c)

This registration is achieved for a very large amplitude of apparent displacement field in both $x$ and $y$ directions, as shown in Figures 3(d) and 3(e) The most salient property of the displacement field is the fact that the $x$ axis 
is more or less uniformly stretched by $1 \%$ whereas the $y$ axis shows a similar uniform contraction of about $-1 \%$. This observation suggests that pixels are in fact elongated to rectangles along the slow scan direction, whatever the scan direction $\left(0^{\circ}\right.$ or $\left.90^{\circ}\right)$. To get a more precise appreciation of these distortions, it is interesting to compute the strains from the apparent displacement field, as shown in Figures $3(\mathrm{f})] 3(\mathrm{~g})]$ and $3(\mathrm{~h})$, $\epsilon_{x x}$ and $\epsilon_{y y}$ show marked horizontal and vertical stripes respectively, while a 'tartan' fabric pattern is visible in $\epsilon_{x y}$ field. In agreement with the previous observation, the dominant strain is a stretch (respectively contraction) of about $1 \%$ along the $x$ (resp. the $y$ ) direction, as can be seen from the average values of the corresponding strain components. 


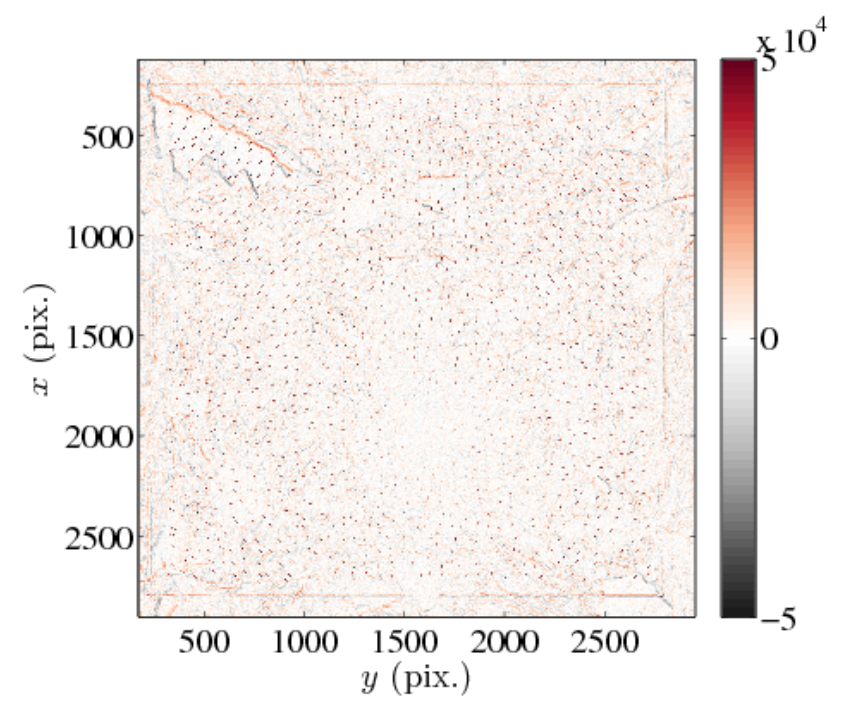

(a) Initial difference

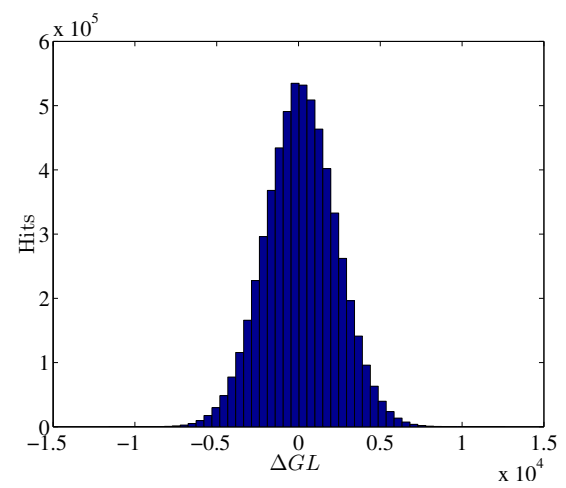

(c) Histogram of residual

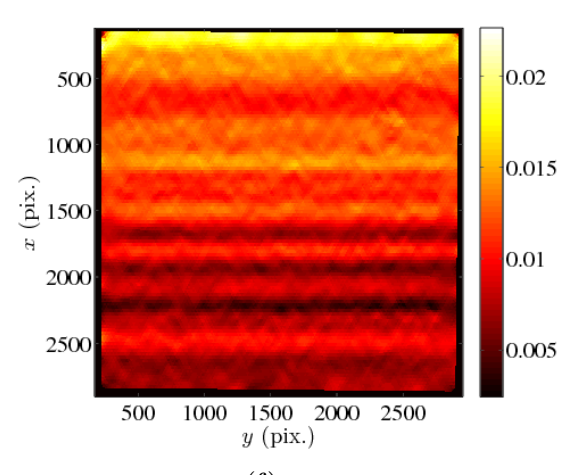

(f) $\epsilon_{x x}$

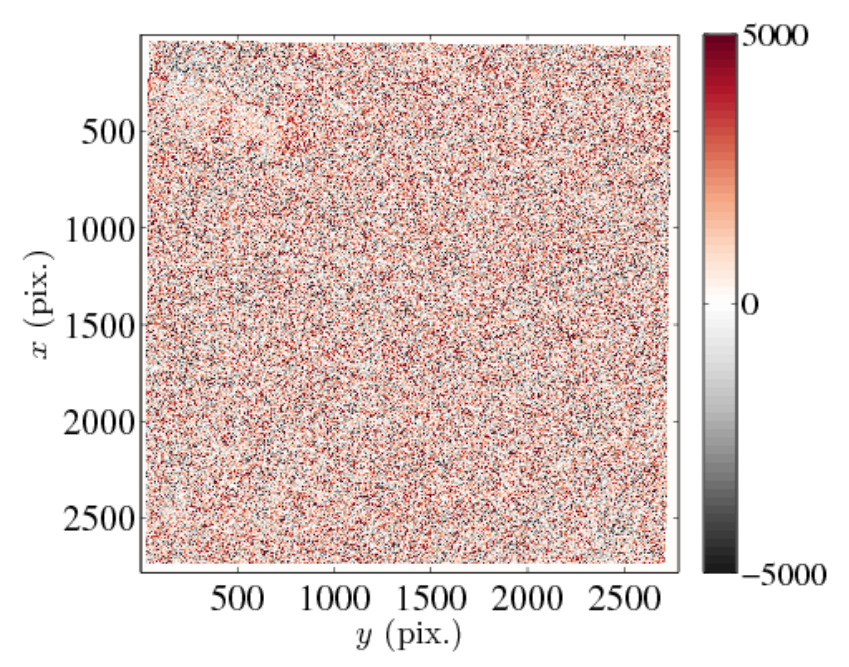

(b) Final residual

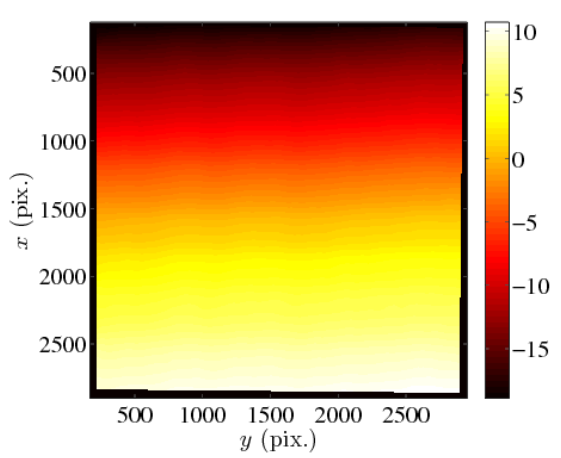

(d) $U_{x}$

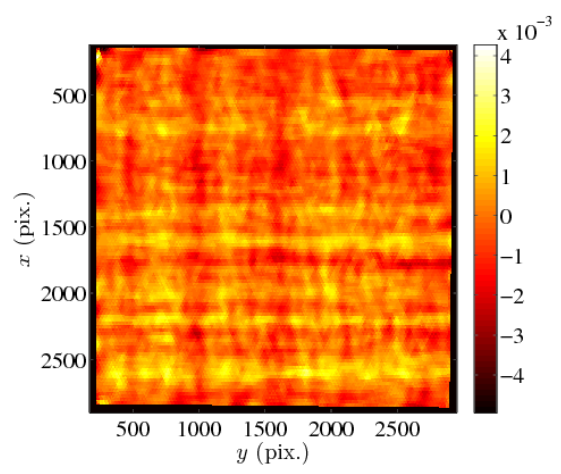

(g) $\epsilon_{x y}$

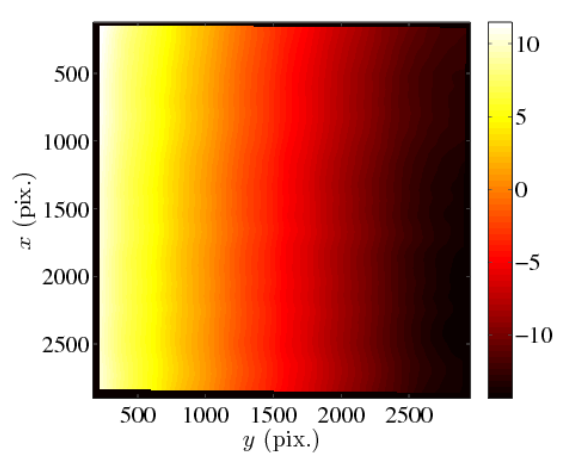

(e) $U_{y}$

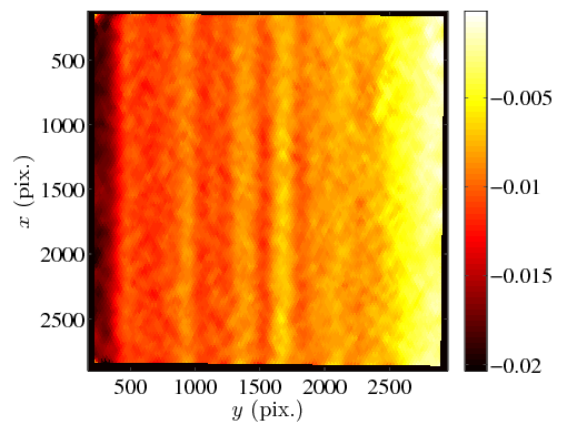

(h) $\epsilon_{y y}$

Figure 3: DIC results for BSE images of the reference state acquired with rotated $0^{\circ}$-scan and $90{ }^{\circ}$-scan (Figure 2). (a) Initial difference between the two images. (b) Corresponding residual field at convergence. A 'grid' like pattern is visible. (c) Histogram of the gray level residual. (d,e) Displacement field in $x$ and $y$ directions (expressed in pixels). (f-h) Strain fields. The pixel size is $42 \mathrm{~nm}$ for all images

The remarkable and specific patterns exhibited by the strain field can be explained by the fact that SEMs control the electron beam more precisely in the fast scan direction, while the position of the starting point of the next scanned line is less accurate. Consequently, the spacing error between scanned lines induces bands in $\epsilon_{x x}$ and $\epsilon_{y y}$ fields and the incorrect alignment leads to the grid in $\epsilon_{x y}$ field. Following Ref. [13, it is proposed to combine images acquired with different scan directions so that each fast-scan line is preserved untouched but may be repositioned at a slightly corrected starting point. This procedure, which revealed successful for AFM images, 
is here applied to register the above different scan orientation images.

\subsection{SEM scan correction}

The correction procedure is based on the hypothesis that the fast-scan direction is correct in SEM images 13 . Let $\phi(\boldsymbol{x})$ be the undistorted image, which is unknown. Image $f_{1}$ is captured with a fast-scan direction along the $x$-axis, and image $f_{2}$ captured along the $y$-axis. Thus each line $f_{1}(., y)$ and $f_{2}(x,$.$) is considered exact. For the$ first image, when the incident beam moves to the start position of the next line, it is assumed that a repositioning error exists with components $u_{x}$ and $u_{y}$ in the sample surface plane. The two components $u_{i}$ of the displacement are assumed to only depend on the slow scan direction $y$. Similarly for image 2, each line is repositioned with a displacement $\boldsymbol{v}(x)$. This is expressed as

$$
\begin{aligned}
& f_{1}[\boldsymbol{x}+\boldsymbol{u}(y)]=\phi(\boldsymbol{x}) \\
& f_{2}[\boldsymbol{x}+\boldsymbol{v}(x)]=\phi(\boldsymbol{x})
\end{aligned}
$$

where $\boldsymbol{u}=\left(u_{x}, u_{y}\right)$ and $\boldsymbol{v}=\left(v_{x}, v_{y}\right)$ are two unknown vector profiles.

The objective of the procedure is to estimate $\phi$ from $f_{1}$ and $f_{2}$ as well as the displacements $\boldsymbol{u}$ and $\boldsymbol{v}$. Equation (2) belongs to the global DIC techniques, with the specific difficulty of unknown reference $\phi$. Figure 4 shows the correction results for the undeformed state. The displacement was decomposed over 50 Fourier modes (this is an arbitrary choice but other discretizations provide very similar results). The analysis requires less than 2 hours for $3072 \times 3072$-pixel images on a PC with i7 CPU and 32 Gb of memory.

Figure $4(\mathrm{~g})$ shows the residual field of the slow-scan error correction procedure. No original image features are clearly apparent and hence the registration procedure is deemed trustworthy. It is worth noting that residuals are very significantly decreased as compared to the previous approach, because the effect of artifacts is more precisely formulated. Figures 4(a) and 4(b) show the $x$ and $y$ components of the displacement fields $\boldsymbol{u}$ between image $f_{1}(\boldsymbol{x})$ and the best achieved compromise $\phi(\boldsymbol{x})$.

Figure 4(c) shows $\partial_{x} u_{x}$, which is comparable to $\epsilon_{x x}$ shown in Figure $3(\mathrm{f})$. Similarly, Figures 4(d) and 4(e) show the $x$ and $y$ components of the displacement field $\boldsymbol{v}$ between image $f_{2}(\boldsymbol{x})$ and $\phi(\boldsymbol{x})$. Figure $4(\mathrm{f})$ shows $-\partial_{y} v_{y}$, which is comparable to $\epsilon_{y y}$ shown in Figure $3(\mathrm{f})$. Under a small strain assumption, the difference between these two fields, $\boldsymbol{u}-\boldsymbol{v}$, should be comparable to the previous standard DIC displacement field shown in Figure 3. It is observed that the agreement is quite satisfactory. All the bands in the strain field are well captured by the slow-scan error correction procedure. This result validates the previous DIC approach, but also indicates that the present approach is more reliable (i.e., showing smaller residual levels). The mean gradients of $\boldsymbol{u}$ and $\boldsymbol{v}$ are respectively $1.12 \%$, and $1.07 \%$, in very good agreement with the previous determination of about $1 \%$ mismatch between fast and slow scan directions. 


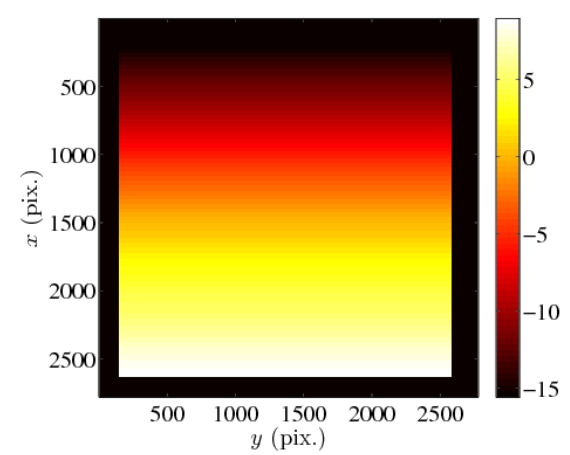

(a) $u_{x}$

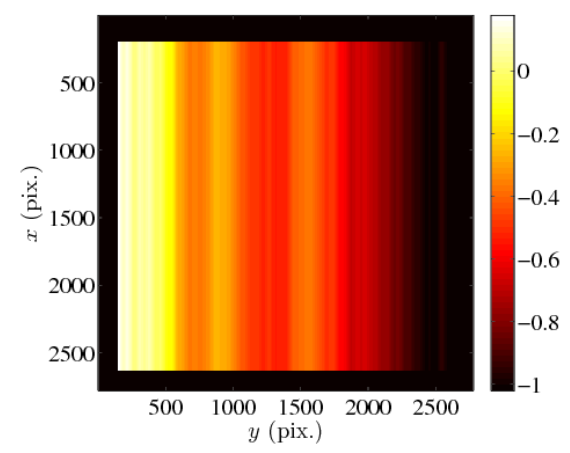

(d) $v_{x}$

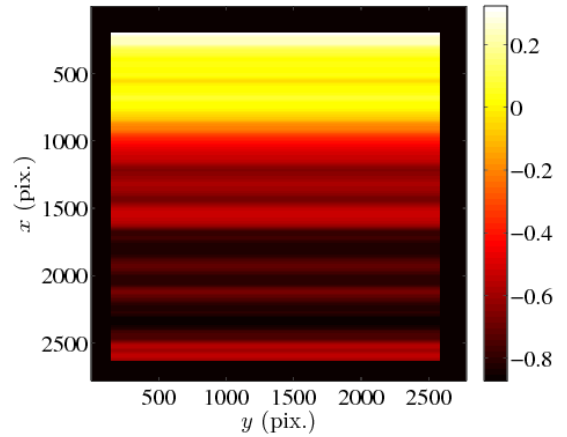

(b) $u_{y}$

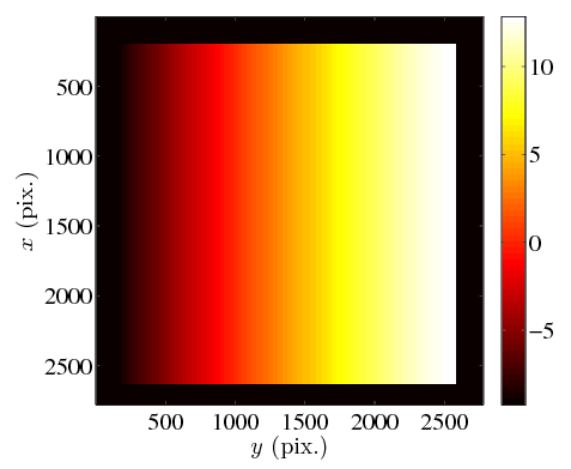

(e) $v_{y}$

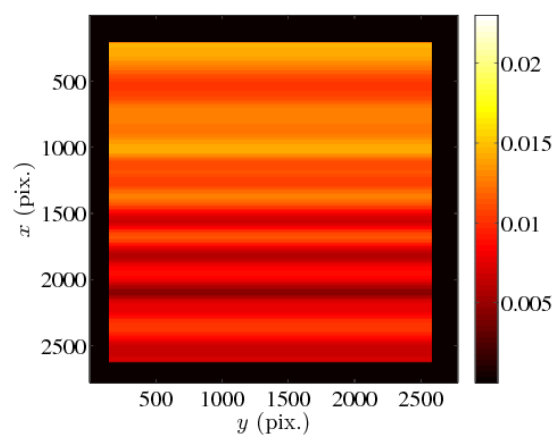

(c) $\partial u_{x} / \partial x$

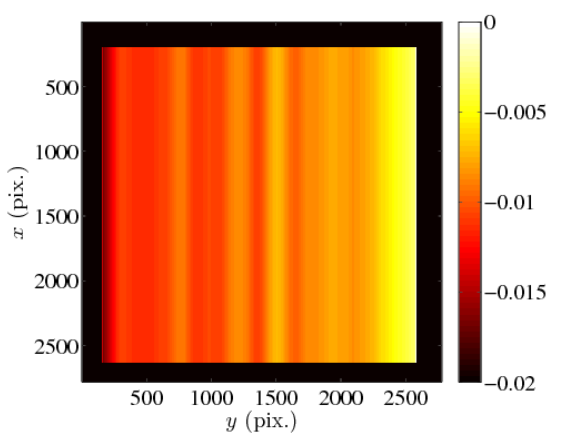

(f) $-\partial v_{y} / \partial y$

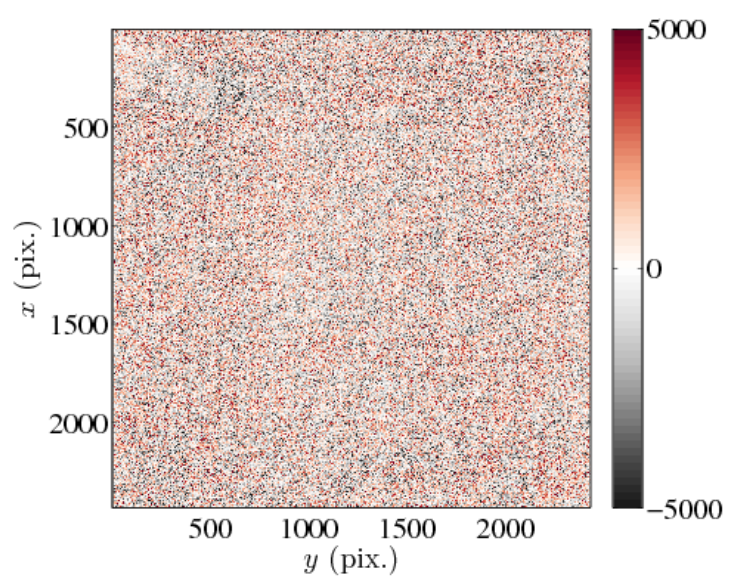

(g)

Figure 4: DIC results for the correction of BSE images $f_{1}$ and $f_{2}$ for the initial state. (a-b) Displacement field in $x$ and $y$ directions between image $f_{1}$ and the corrected image. (c) Gradient of figure (a) in $x$ direction. (d-e) Displacement field in $x$ and $y$ directions between image $f_{2}$ and the corrected image. (f) Gradient of figure (e) in $y$ direction. (g) Residual field $f_{1}(\boldsymbol{x}+\boldsymbol{u})-f_{2}(\boldsymbol{x}+\boldsymbol{v})$. The pixel size is $42 \mathrm{~nm}$ for all images

The above procedure confirms the existence of scan drift in SEM images, although less marked than for AFM acquisitions 13. This effect should be taken into consideration if accurate strain and displacement measurements are sought, but at the extra cost of a double acquisition along two perpendicular scan directions. However, it should be noted that the resulting error in measuring strains is the product of the true strain and the spurious one due to scanning. In the present case, accepting a relative $1 \%$ inaccuracy in the local strain, i.e., of order $6 \times 10^{-4}$ uncertainty, one can consider that the BSE images can be trusted.

DIC has been run on all BSE images acquired during the in situ tensile test and the change of $\epsilon_{x x}$ strain is shown in Figure 5 and $\epsilon_{y y}$ in Figure 6. Finite deformation theory has been adopted and the strain tensor is computed as the Green-Lagrange tensor where $y$ is the tensile direction. From $\epsilon_{x x}$ and $\epsilon_{y y}$ changes it is concluded that the strain concentration/localization patterns revealed at the onset of plasticity do not change subsequently. This trend is 
confirmed by previous work on 304L stainless steel 23 . The strain patterns are also consistent with slip traces at the specimen surface, inclined at $45^{\circ}$ with respect to the loading direction.

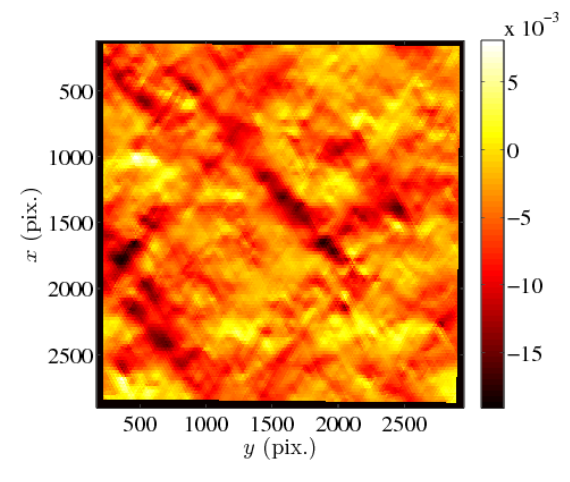

(a) $\left\langle\epsilon_{y y}\right\rangle=0.9 \%$

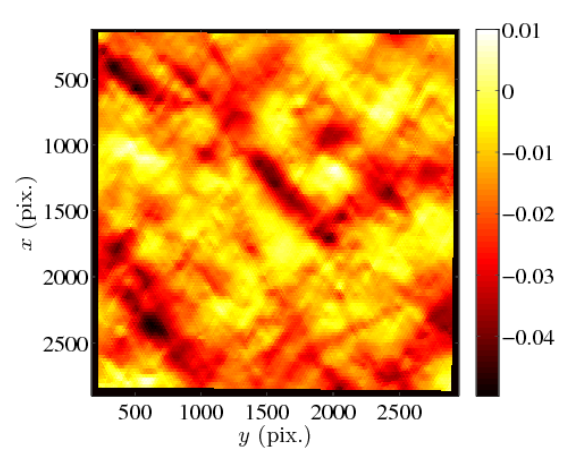

(b) $\left\langle\epsilon_{y y}\right\rangle=2.4 \%$

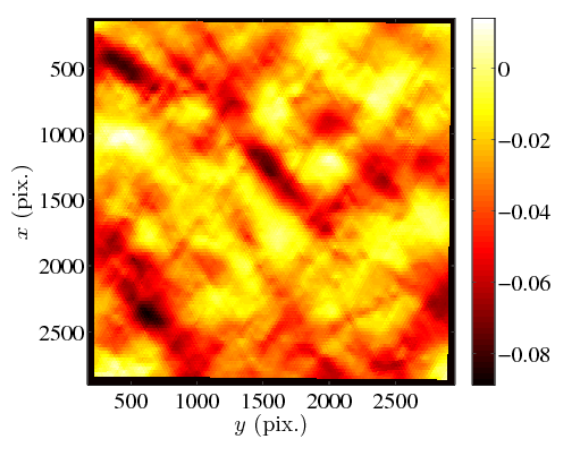

(c) $\left\langle\epsilon_{y y}\right\rangle=5.5 \%$

Figure 5: $\epsilon_{x x}$ fields calculated by DIC on the BSE images acquired during the tensile test for different macroscopic strain levels $\left\langle\epsilon_{y y}\right\rangle$. The pixel size is $42 \mathrm{~nm}$ for all images

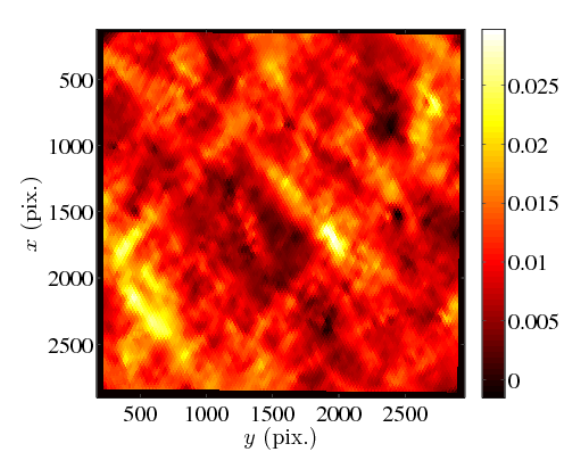

(a) $\left\langle\epsilon_{y y}\right\rangle=0.9 \%$

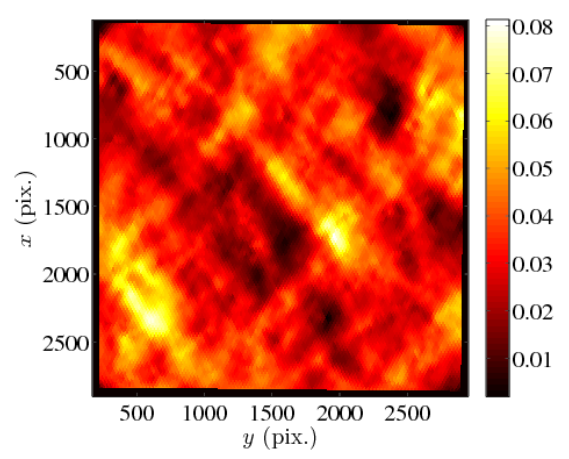

(b) $\left\langle\epsilon_{y y}\right\rangle=2.4 \%$

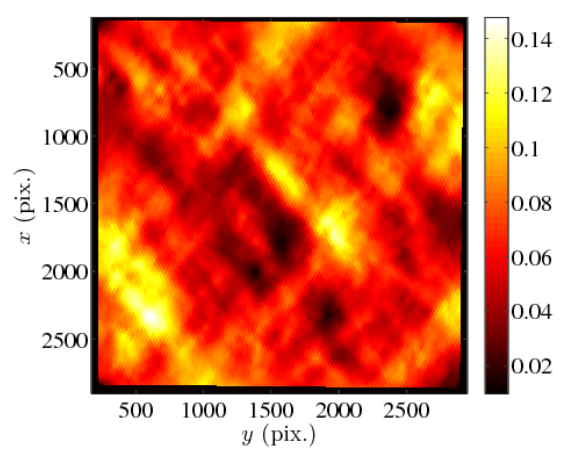

(c) $\left\langle\epsilon_{y y}\right\rangle=5.5 \%$

Figure 6: $\epsilon_{y y}$ fields calculated by DIC on the BSE images acquired during the tensile test for different macroscopic strain levels $\left\langle\epsilon_{y y}\right\rangle$. The pixel size is $42 \mathrm{~nm}$ for all images

\section{Treatment of EBSD images}

Recently, quaternion correlation has been proposed to measure crystal rotation and displacement fields [5]. The algorithm uses the crystallographic orientation maps provided by EBSD images as registration support and the residual field indicates the rotation field. The algorithm leads to displacement fields with sub-pixel uncertainty and the rotation field with an uncertainty on the order of $10^{-3}$ radian, well below the orientation indexation error/resolution of EBSD.

The orientation maps of 4 EBSD acquisitions are shown in Figure 7f Figure 7(c) shows the IQ field corresponding to Figure 7(a). The square encompassing speckles is distorted, thereby indicating a systematic drift of EBSD acquisition, a phenomenon that will be addressed in Section 6. It is worth noting that two EBSD acquisitions might use different reference orientations, which will result in non-sensical disorientation through quaternion correlation even after the application of crystal symmetry. To correct this, one can choose arbitrarily a set of Euler angles from the same region in the two acquisitions, say those at the barycenter of the largest grain in the area, and transform them into a fixed interval, say $\left(\varphi_{1} \in[0, \pi], \varphi \in[0, \pi / 2], \varphi_{2} \in[0, \pi / 2]\right)$, through crystal symmetry. Then the difference of their reference becomes clear and easily cancelled. The reference difference calculated from the two selected pixels can be applied for the entire region, because during an acquisition procedure the reference orientation does not change and the reference difference is universal for two given EBSD acquisitions. 


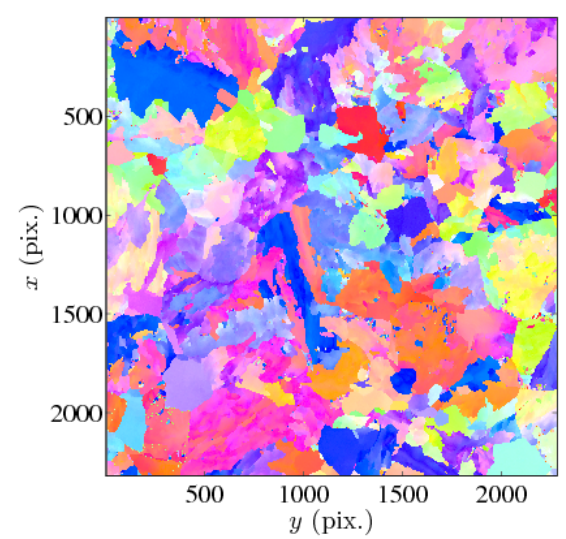

(a)

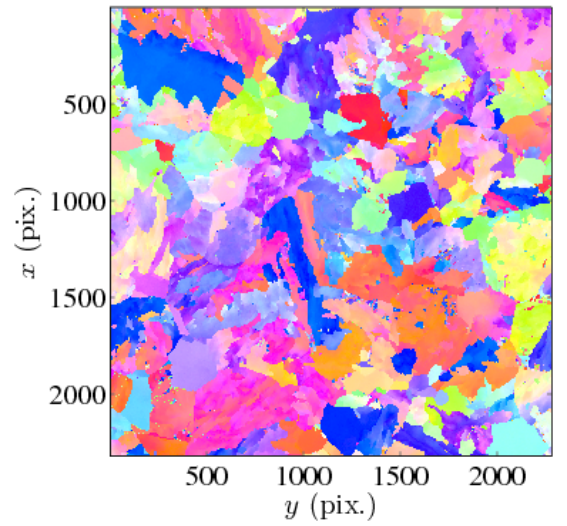

(b)

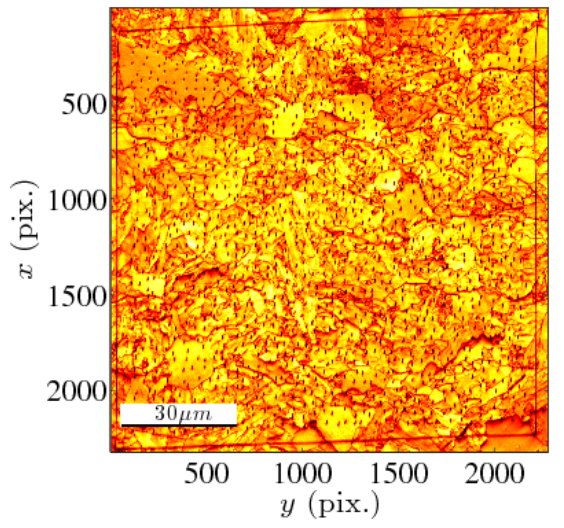

(c)

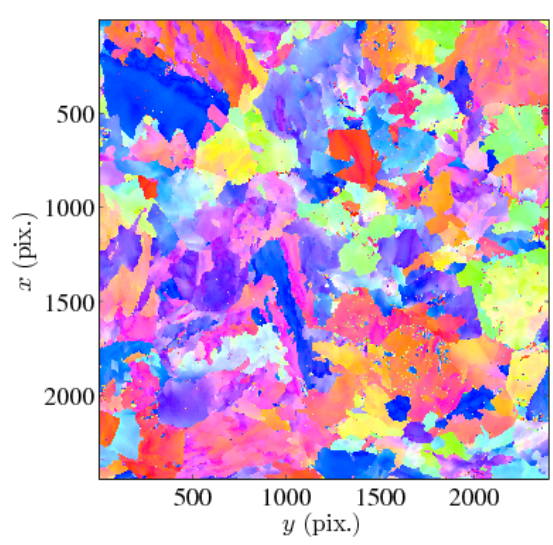

(d)

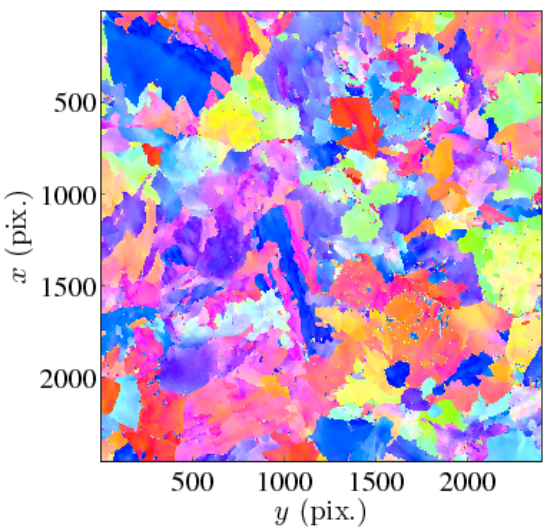

(e)

Figure 7: Orientation maps of successive EBSD acquisitions of the virgin sample (a,b), corresponding IQ map (c). Orientation maps of the deformed sample $(\mathrm{d}, \mathrm{e})$. The pixel size is $50 \mathrm{~nm}$ for all images

The difference between successive acquisitions (without any difference in loading) is not visible to the naked eye, and hence, they were analyzed using quaternion correlation. The DIC parameters for quaternion correlation and IQ field correlation are: i) an unstructured triangular mesh with element size 25 pixels; ii) the mechanical regularization length is brought down gradually from 400 to 200 and 100 pixels, each time initialized with the results of the last calculation; iii) the displacement field obtained by IQ field correlation is used as initialization for quaternion correlation, to save computation time. The results provided by quaternion correlation reveal non negligible disorientations and the displacement field provided by IQ field correlation show spatial inconsistencies, as shown in Figures 8 and 9 .

It can be seen that the displacement fields in $x$ and $y$ directions have a range of 11 pixels and 22 pixels, respectively, for the undeformed sample, 90 pixels and 50 pixels for the deformed sample. Significant displacement gradients are visible at the top, indicating severe instabilities in the beginning of EBSD acquisition. In addition, horizontal traces appear in both $x$ and $y$ directions, which means the slow scan direction drift studied in Section 4 is also present in EBSD acquisitions (presumably because of the long duration of the acquisition overnight). The large uncertainty in location must be taken into consideration during the exploitation of EBSD results.

The amplitude of disorientation and the corresponding rotation vector components are shown in these figures. It is seen that a tilted thick band of low disorientation zone, i.e., less than $0.5^{\circ}$, exists even though no load was applied (Figure 8(c)]. The corresponding rotation vector components $\omega_{x}, \omega_{y}$ and $\omega_{z}$ are noisy in the band. This is in accordance with the small magnitude of rotation that renders the rotation vector ill-defined. However, outside the band the disorientation level increases very significantly, reaching about $1.8^{\circ}$ in the corner. The rotation vector $\boldsymbol{\omega}$ shows a tendency, too. For example, Figure 8(e) shows a high level of $\omega_{y}$ at the top left corner and low level at the down right corner. 


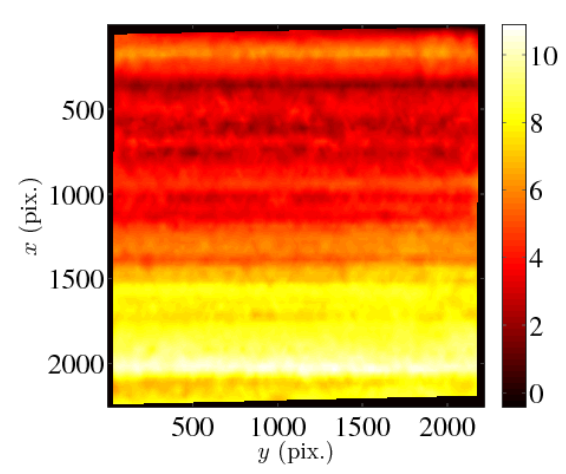

(a)

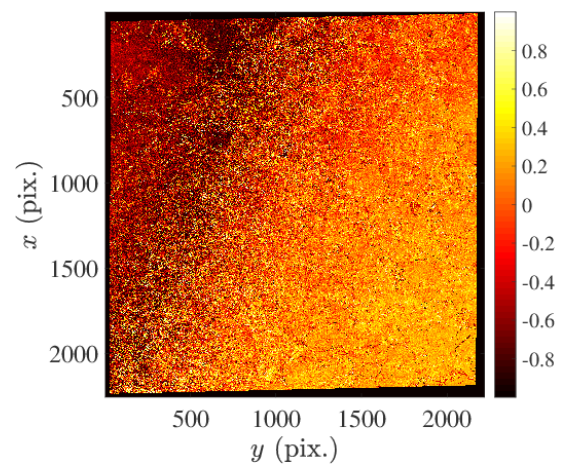

(d)

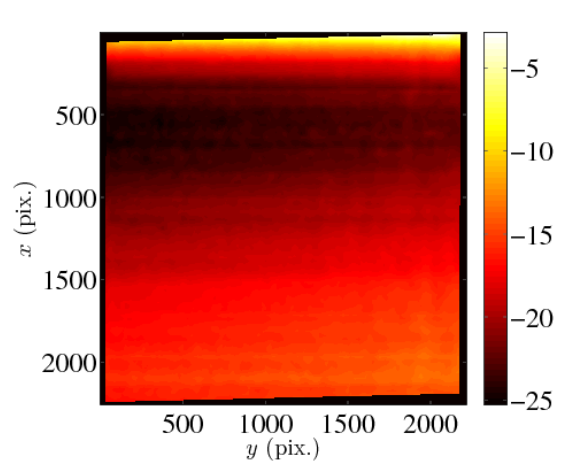

(b)

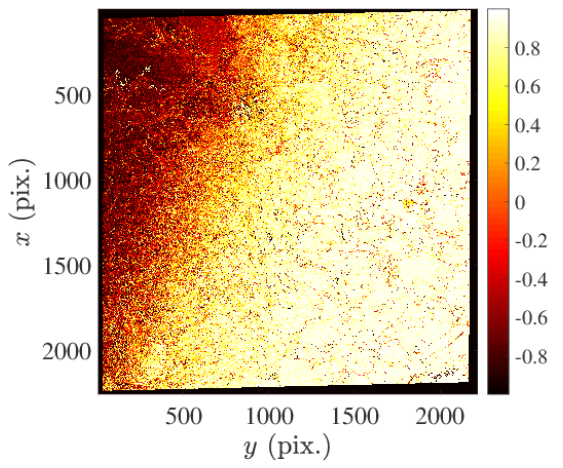

(e)

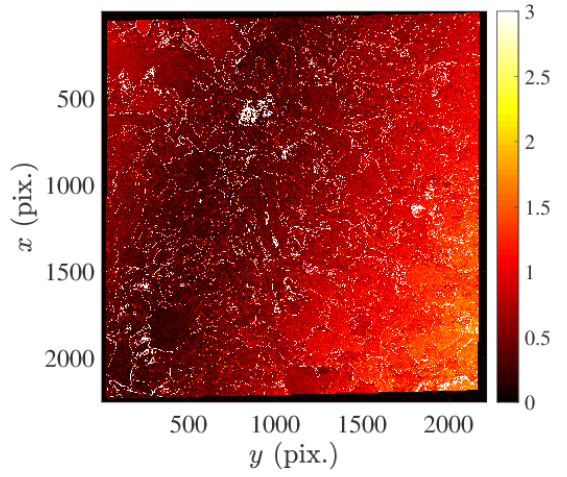

(c)

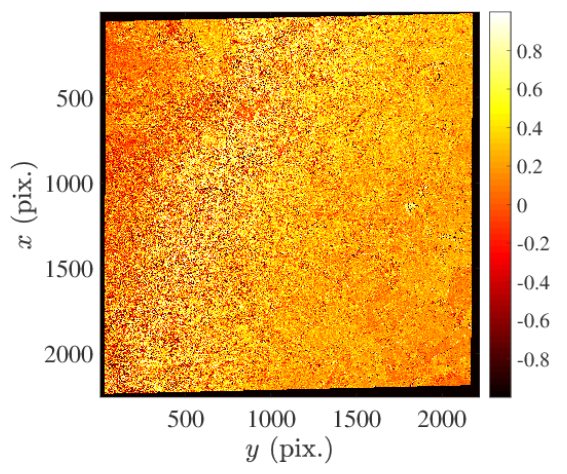

(f)

Figure 8: DIC results between successive EBSD acquisitions (virgin sample). (a-b) Displacement field in $x$ and $y$ directions respectively. (c) Disorientation angle (in $\left.{ }^{\circ}\right)$; (d-f) 3 components of disorientation vector $\omega_{x}, \omega_{y}$ and $\omega_{z}$, respectively. The pixel size is 50 nm 


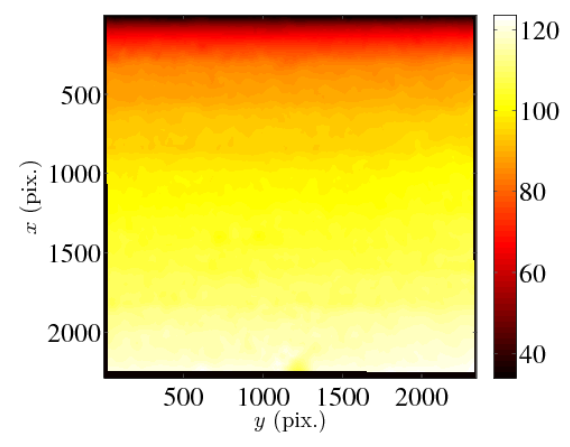

(a)

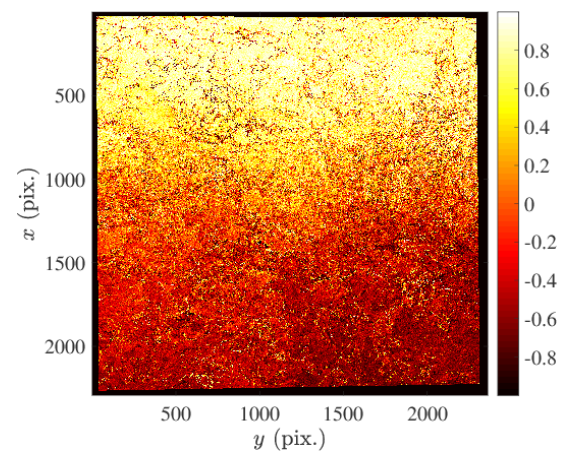

(d)

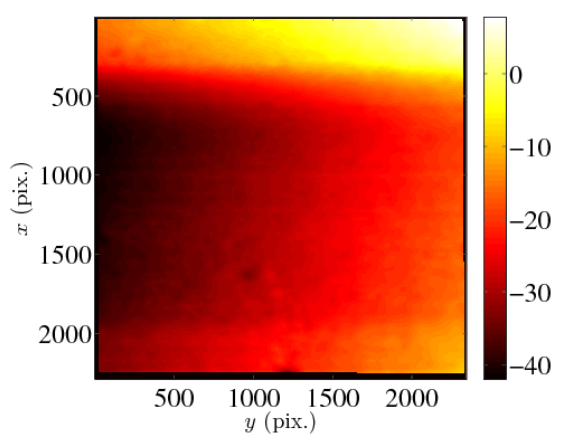

(b)

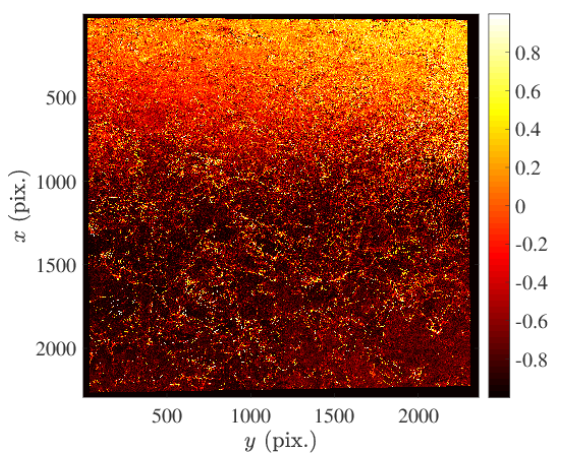

(e)

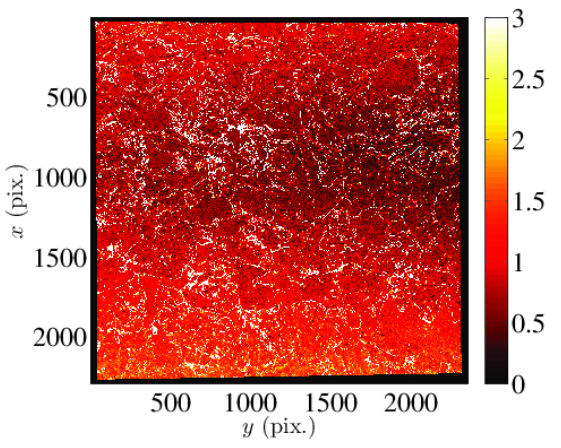

(c)

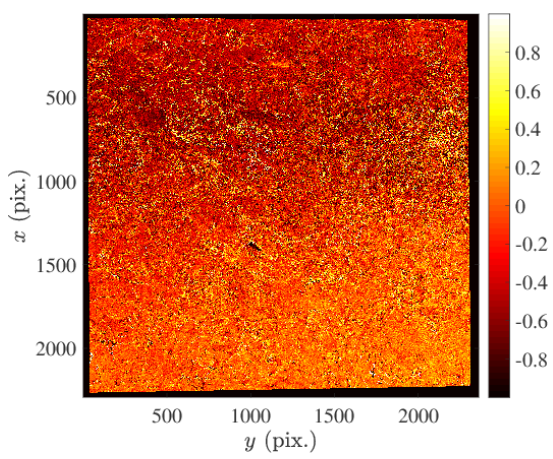

(f)

Figure 9: DIC results between successive EBSD acquisitions (deformed sample). (a-b) Displacement field in $x$ and $y$ directions respectively. (c) Disorientation angle (in ${ }^{\circ}$ ). (d-f) 3 components of disorientation vector $\omega_{x}, \omega_{y}$ and $\omega_{z}$, respectively. The pixel size is $50 \mathrm{~nm}$

Further, grain shapes are revealed by the fluctuations of the components of $\boldsymbol{\omega}$, and not by the disorientation. In other words, the disorientation angle between two successive scans is not grain-specific, yet the disorientation vector is. Satisfactory explanations are not available yet. Several possible reasons may be proposed: i) the sample has a thermal distortion between two scans, either by heat induced by the electron beam, or due to the room temperature differences between the two days of the experiment. ii) Dynamic focus does not work perfectly. As during the experiment, dynamic focus is performed on the image center, the electron beam is not well focused for the upper and lower parts, as the working distance may vary. This phenomenon explains the horizontal band in Figure 9, but not the tilted band in Figure 8, iii) Physical drift of the imaging device, namely, either the sample support drifts during acquisitions, or the EBSD device is sucked firmly into the chamber by vacuum during the two-day acquisitions. As a result, the indexed orientation is to be used with caution. However, as the macroscopic tensile strain level is $6 \%$ and significant crystal rotations occur, the uncertainty associated with such smooth spatial variations with a maximum level of $1.8^{\circ}$ will not be considered as a severe problem.

Quaternion correlation has been run to study crystal rotations during the tensile test. However, as quaternion correlation is based on the hypothesis that the crystal orientation and grain boundaries do not change significantly [5], which is not the case herein, a precise initialization is needed. A global and regularized DIC procedure has been successfully applied to grain boundary images to obtain an initial displacement field. A regular triangular mesh with characteristic length of 30 pixels is chosen and mechanical regularization length is reduced gradually from 200 to 70 pixels 34 . The grain boundary images and the corresponding displacement field are shown in Figure 10 


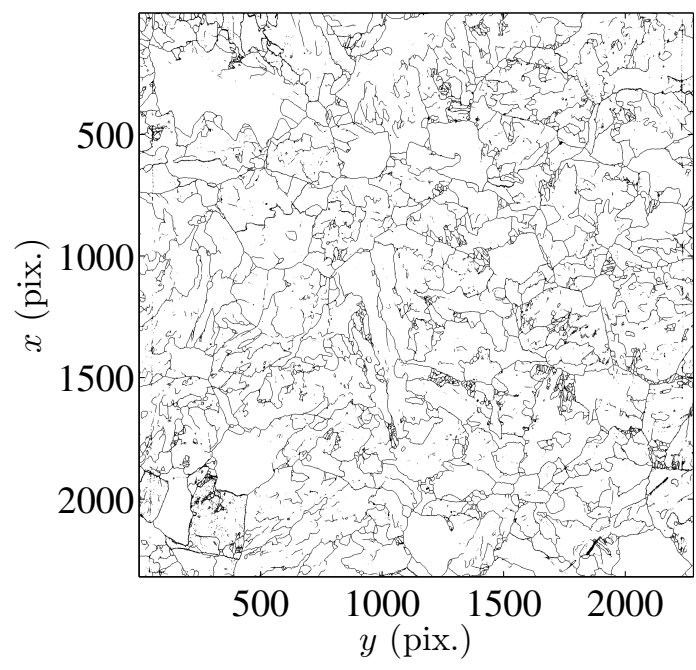

(a)

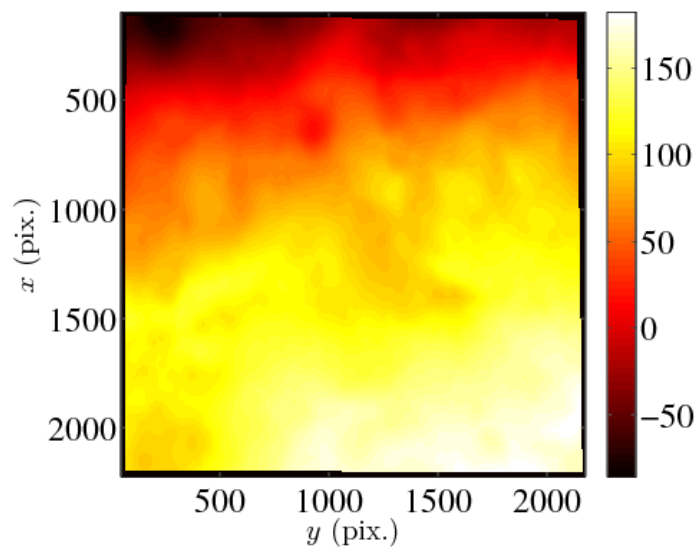

(c)

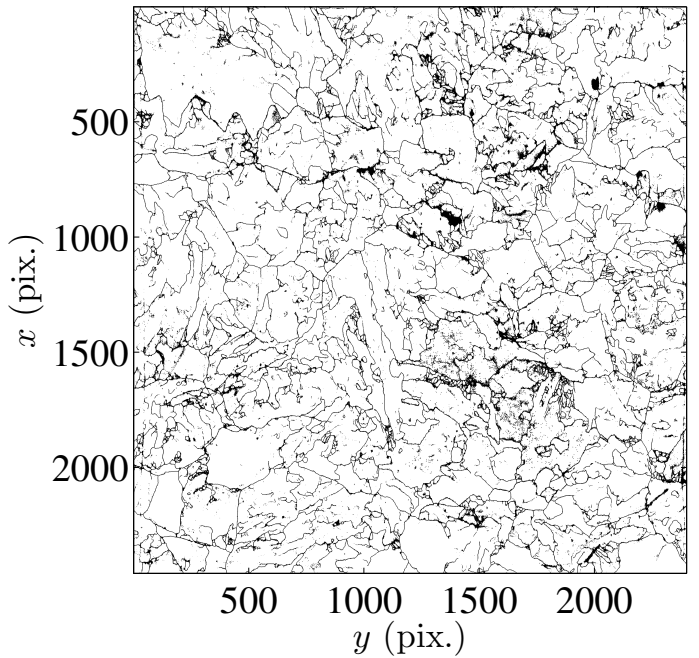

(b)

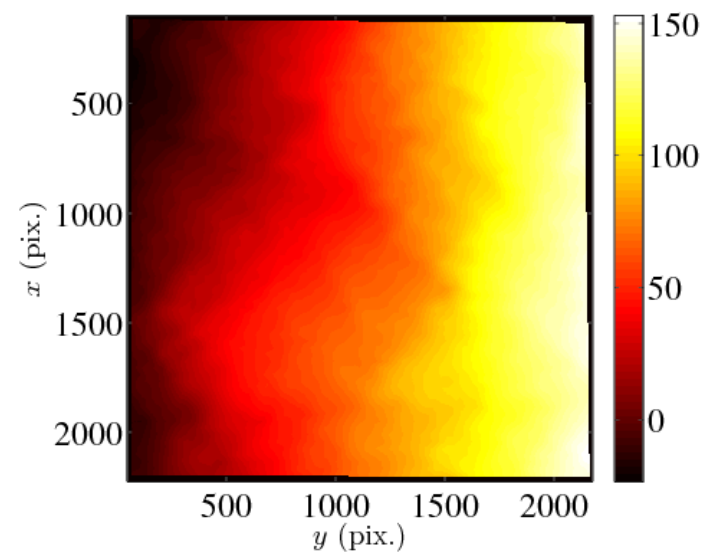

(d)

Figure 10: DIC results on grain boundary images. (a) Reference picture corresponding to Figure 7(b) (b) Deformed picture corresponding to Figure $7(\mathrm{e})$ (c-d) Resulting $u_{x}$ and $u_{y}$ displacement fields, respectively. The physical size of one pixel is equal to $50 \mathrm{~nm}$

This displacement field is used to initialize quaternion correlation, which results in the rotation field shown in Figure 11. It is observed that rotations concentrate significantly, with a maximum level $15^{\circ}$. High gradients exist in several grains. 


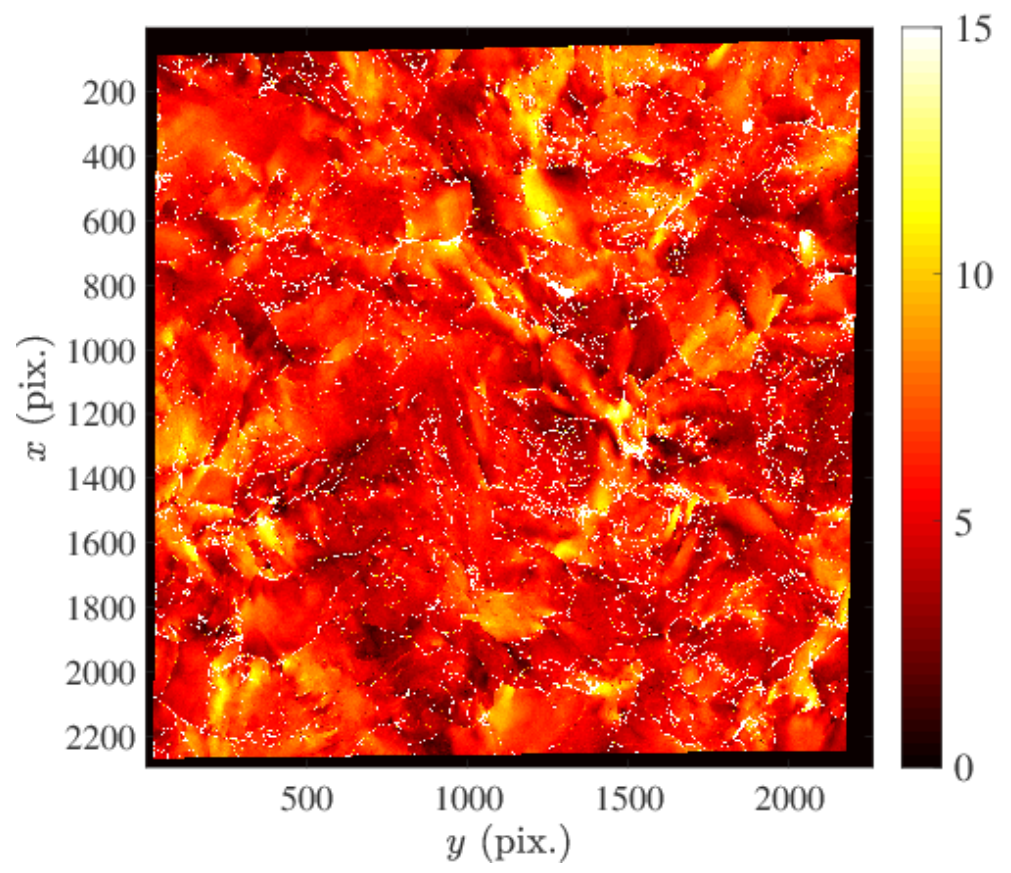

Figure 11: Rotation field (in o) between two EBSD acquisitions before and after the tensile test (Figures $7(\mathrm{~b})]$ and $7(\mathrm{e})]$. The pixel size is $50 \mathrm{~nm}$

Until now the BSE image series has been registered by DIC, and EBSD images by quaternion correlation. However, the matching of these two modality images and its benefits have not been discussed yet and are detailed in the next section.

\section{Registration of EBSD and BSE images}

EBSD images provide the crystal orientation with a standard angular uncertainty of $0.5^{\circ}$, thus accurate enough for describing the microstructure of the sample. As discussed in Section 4, BSE images with fast scan accuracy in both directions can be obtained, so the spatial coordinates of pixels are obtained very precisely. A natural idea is to combine EBSD and BSE images to give a description of the microstructure with the accuracy of crystal orientation of EBSD image and spatial coordinates of BSE images. Manual matching of EBSD and SEM images has been performed in previous works [22, without stating the accuracy of the registration. The matching has also been performed by calculating a transformation matrix $\boldsymbol{F}$ from several markers visible in EBSD and SEM images 8 . The corresponding error has been predicted to be about 15 pixels or $2 \mu \mathrm{m}$ for an area of $400 \times 400 \mu \mathrm{m}^{2}$. Registration can be obtained by correlating the two types of images, thanks to the image contrast introduced by a large number of speckles that are visible in both modalities.

\subsection{Analysis of speckles in EBSD and BSE images}

Figure 12 shows a zone in EBSD (orientation and IQ) and BSE images. It is observed that the speckles are invisible in the orientation map (Figure 12(a)], thus the deposited speckles do not impact the crystal orientation detection/acquisition. For the IQ image, each speckle corresponds to two darker spots (Figure 12(b)). 


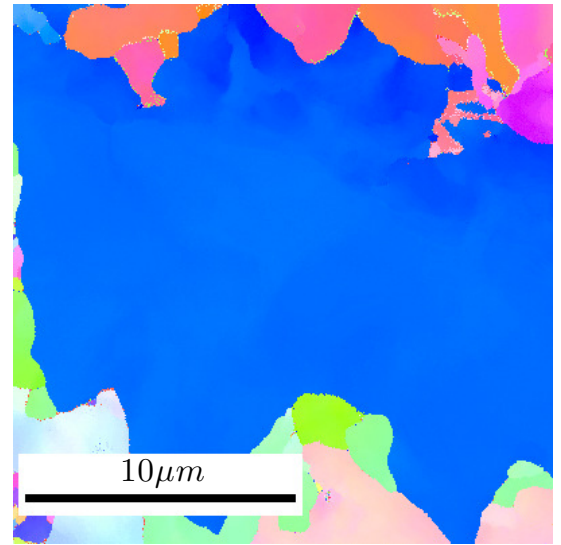

(a)

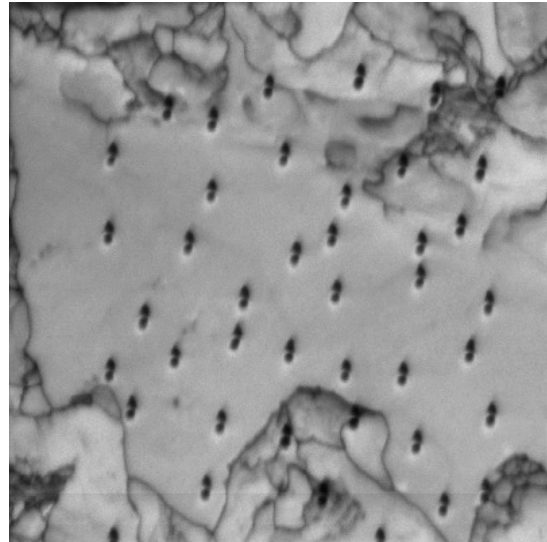

(b)

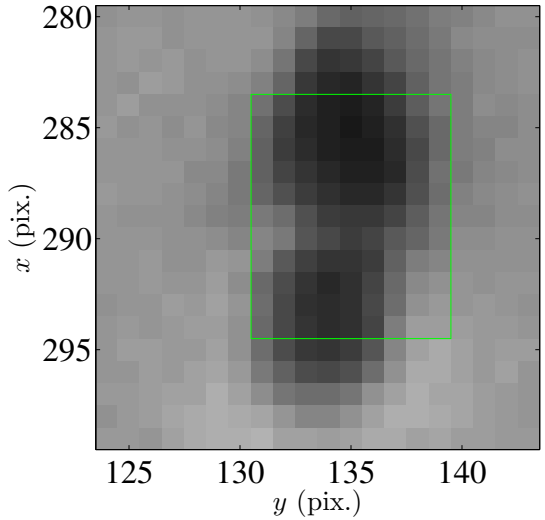

(c)

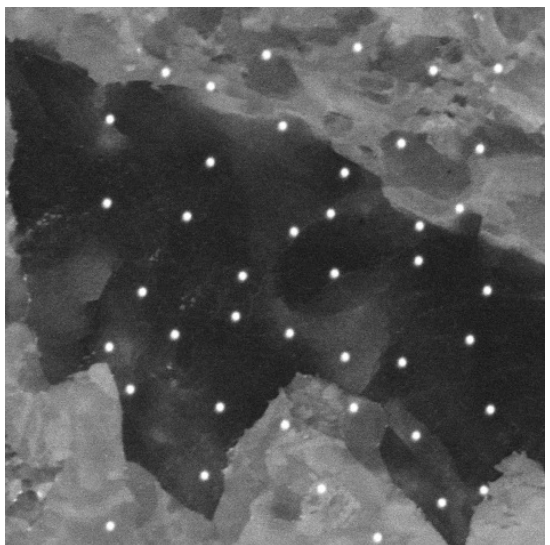

(d)

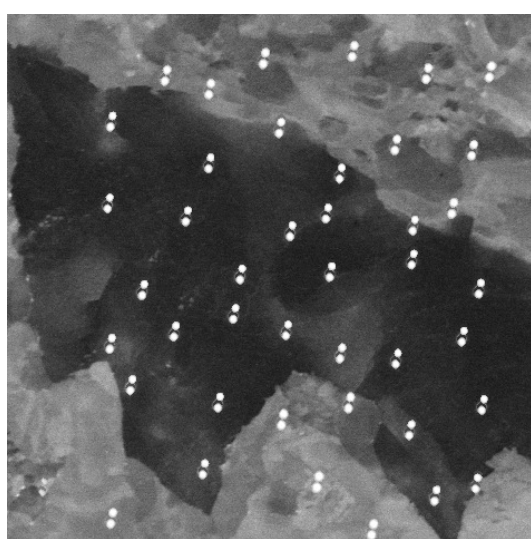

(e)

Figure 12: Several images of the same region of interest of the sample obtained from (a) EBSD orientation map, (b) EBSD IQ image, (d) BSE image, (e) BSE image with artificial 'shadow' speckles. (c) is an enlargement of twin spots shown in sub-figure (b). The diffraction images corresponding to the marked rectangular area are shown in Figure 14

Figure 13 illustrates the formation of the twin spots, which is based on previous studies on the origin of diffraction patterns [37, 38. One spot is linked to the real position of the speckle, the other one is the shadow cast on the sample. When the electron beam hits the speckle (Figure 13(a) its interaction with the underlying material is altered. Thus the diffraction image is deteriorated and so is the image quality of the EBSD analysis. When the electron beam hits a point near a speckle, as shown in Figure 13(b), and if the ballistic range of incident electron is longer than the speckle diameter, the effective back-scattering source is not obscured by the speckle. The diffraction pattern captured by the detector is of better quality than its upper and lower neighbors, thus a higher IQ score between twin spots is obtained. When the electron beam hits the region above the speckle, see Figure 13(c), the speckle with a non negligible height will cast a shadow on the bottom of the diffraction image. 


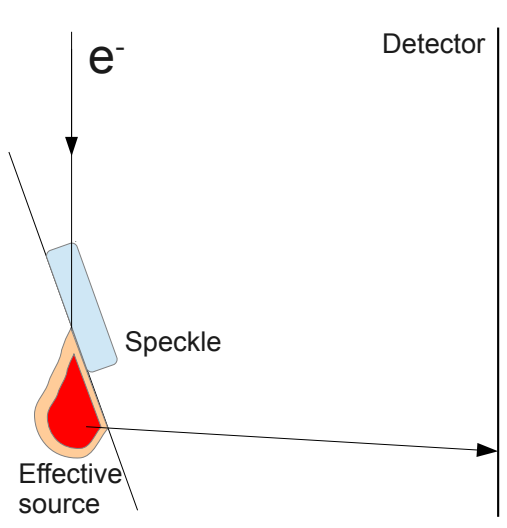

(a)

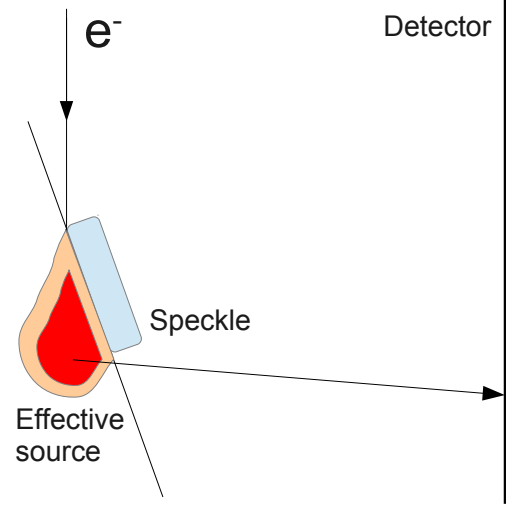

(b)

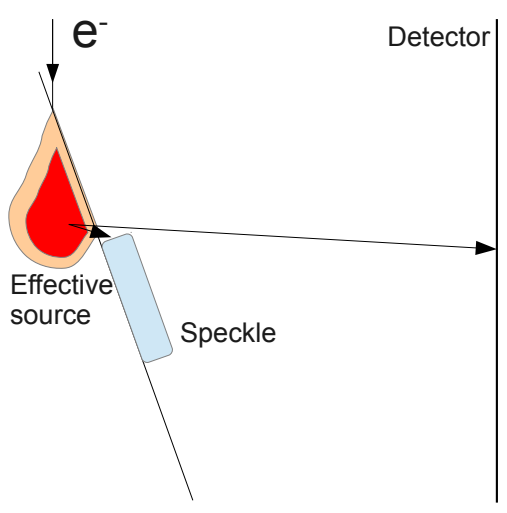

(c)

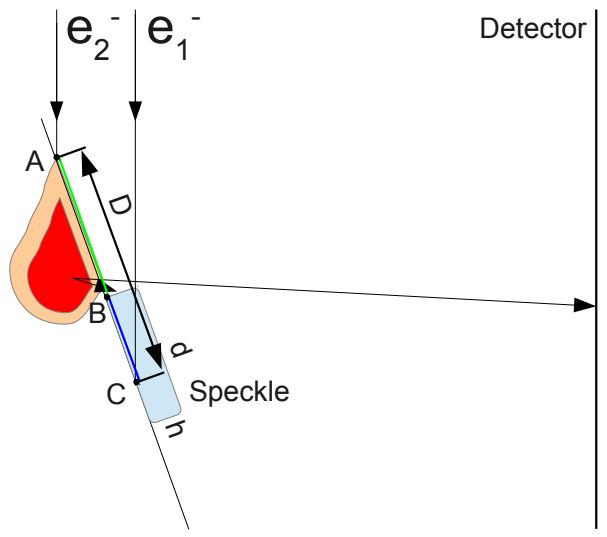

(d)

Figure 13: Schematic sketch providing an explanation to the formation of double spots. (a) Formation of 'real' spot. (b) Formation of 'saddle' between two spots. (c) Formation of shadow spot. (d) Schematic view explaining the distance between the shadow and real spots

As the electron beam moves away, the shadow moves even faster due to the gnomonic projection effect. This schematic explanation is confirmed by the collection of Kikuchi patterns (see Figure 14) recorded as the rectangle shown in Figure $12(\mathrm{c})$ is scanned. It can be seen that for the lower spot a shadow at the bottom of the image appears and vanishes quickly as the electron beam scans through the studied zone. 


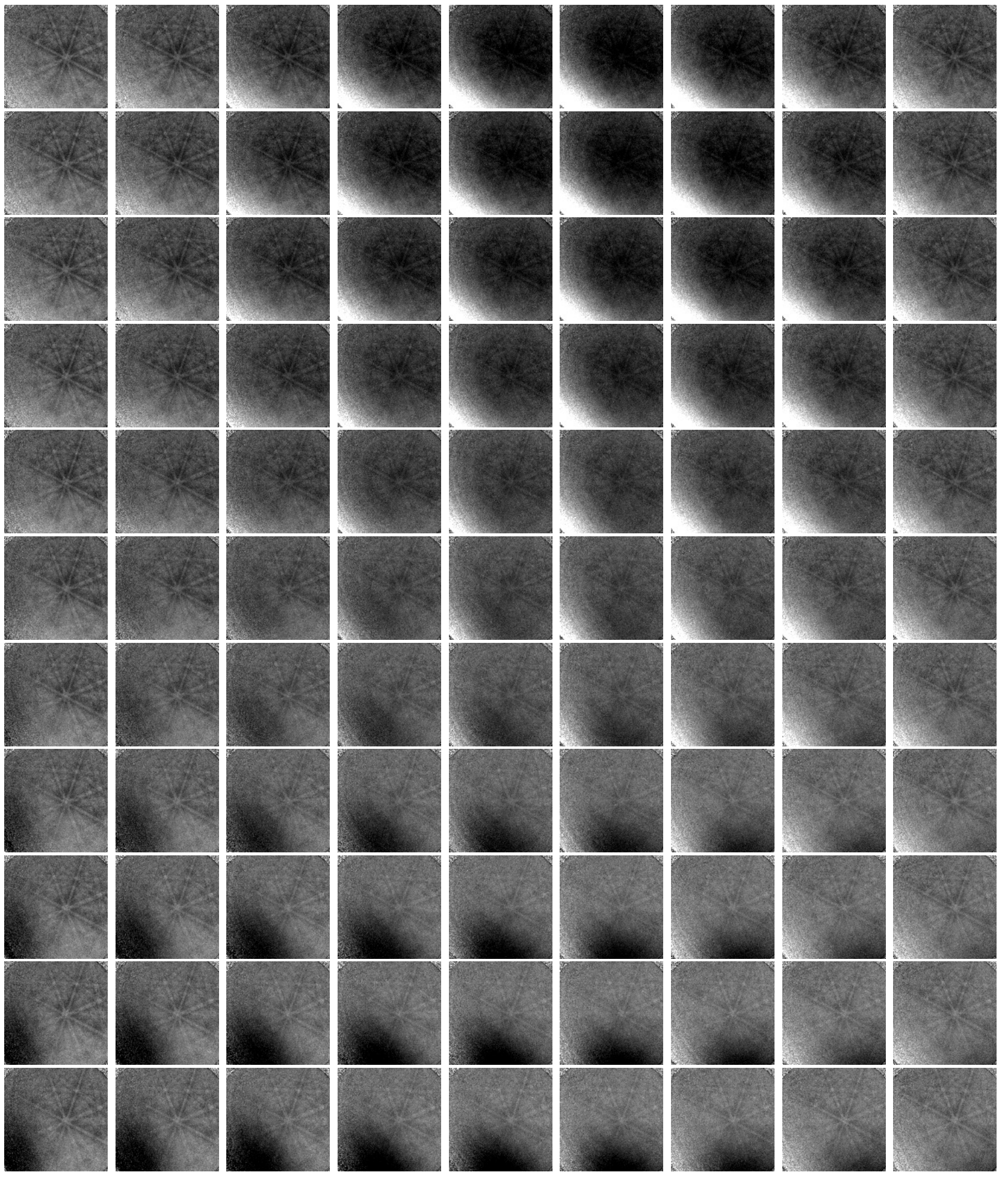

Figure 14: Series of diffraction images of a split speckle. The lower lines of the diffraction images correspond to the shadow spot, as the shadow is visible on the lower edge

As the speckle is a thick disk of $\mathrm{Pt}$ with a diameter much larger than its height, the effective obstacle area is limited. This is the reason why the shadow spot is smaller than the real one. For BSE images, a speckle generates a unique spot (Figure 12(d)). It can be seen from Figure 12(c) that the twin spots are separated by a distance $D$ of about 11 pixels. Figure 13(d) illustrates the origin of this distance. The electron beam $e_{1}^{-}$hits the upper edge of the speckle, thus travels the longest distance inside the speckle and results in the darkest point in the real spot. As for electron beam $e_{2}^{-}$, the most emissive part of the back-scattering electron source is masked by the speckle, and 
the darkest point in the shadow spot is obtained. Consequently,

$$
\begin{aligned}
D & =A B+B C \\
& =A B+\frac{h}{\tan \left(20^{\circ}\right)}
\end{aligned}
$$

where $\boldsymbol{B}$ is the intersection point of the speckle and sample surface, $\boldsymbol{A}$ is the beam hitting point of the darkest point of shadow speckle, $\boldsymbol{C}$ is the beam hitting point of the darkest point of real speckle, and $h$ is the speckle height (i.e., $75 \mathrm{~nm}$ ). Knowing that the pixel size of EBSD acquisitions is $50 \mathrm{~nm}, A B$ is expected to be about 7 pixels, which will be validated in Section 6.2 The fact that $A B$ is larger than the diameter of the speckle in turn validates the analysis of Figure 13(b).

\subsection{Registration results}

The physics behind the 'split' speckles in EBSD IQ images being understood, one should either delete the shadow spot in EBSD IQ image, or create shadow speckles in BSE image if the two types of modalities are to be registered. The latter is chosen herein as shown in Figure 12(e). DIC has been run on the EBSD IQ map and BSE image with shadow speckles, and the results are shown in Figure 15 The coordinates given by EBSD acquisitions are distorted both in $x$ and $y$ directions. More importantly, the distortion is not stable in time, as it is not the same between the two successive EBSD acquisitions and the BSE image, especially in the $y$-direction. It is therefore not possible to characterize once-and-for-all the EBSD scan distortions and use them to correct other EBSD images. Besides, as the displacement field cannot be described by a regular/simple polynomial function, the conventional (i.e., manual) registration of EBSD and SEM images based on the remarkable points in both images and global polynomial interpolation is not very accurate.

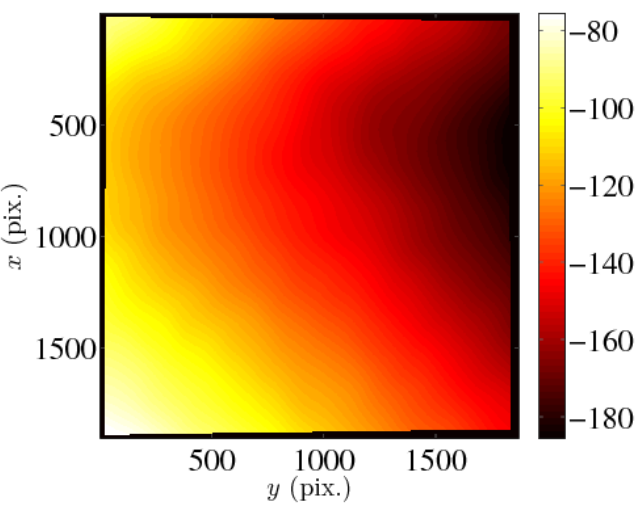

(a)

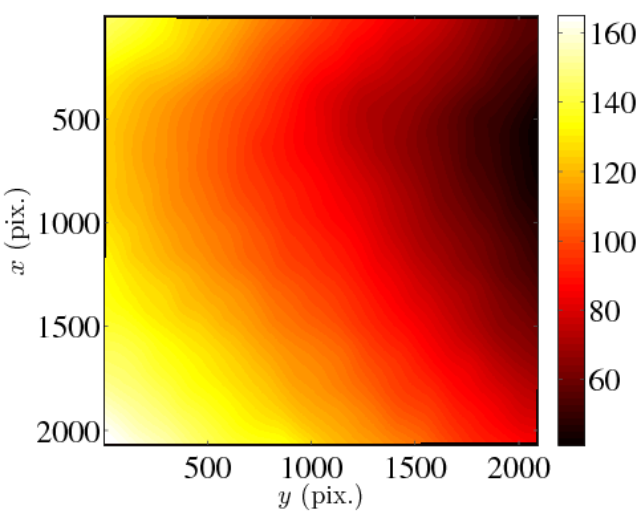

(c)

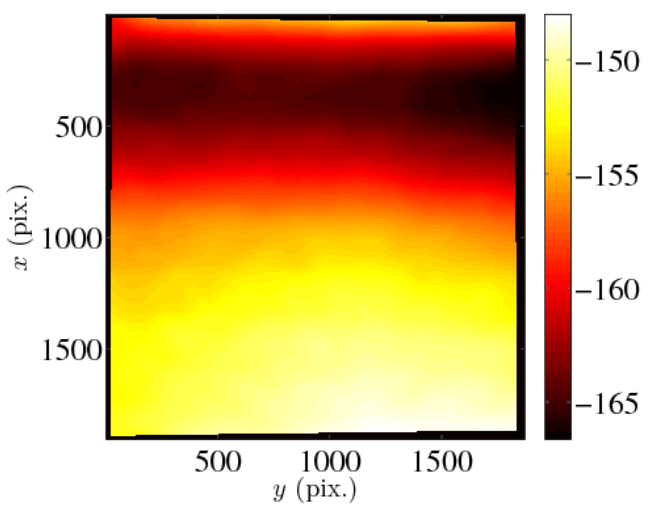

(b)

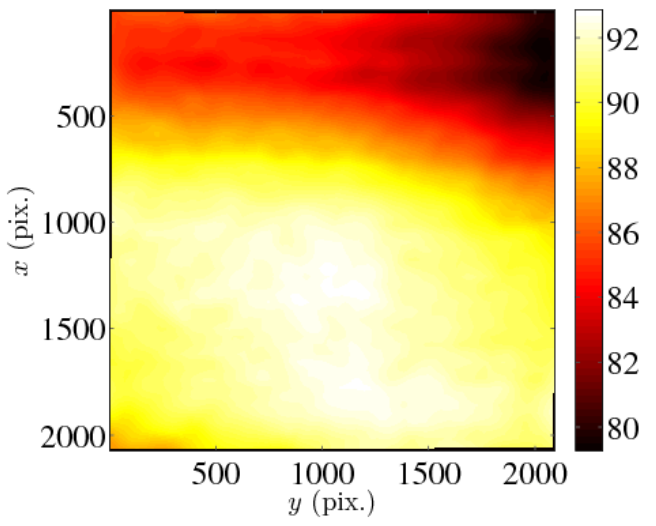

(d)

Figure 15: Correlation results between reference BSE image and first EBSD IQ map. (a) $u_{x}$ (b) $u_{y}$ expressed in pixels. Correlation results between reference BSE image and second EBSD IQ map. (c) $u_{x}$ (d) $u_{y}$ expressed in pixels. The physical size of one pixel is $50 \mathrm{~nm}$ 
As mentioned in Section 4, the corrected BSE image is free from scan drift. Once BSE and EBSD images are registered, accurate spatial coordinates given by BSE images are combined with crystallographic orientations provided by EBSD. Figure 16 shows the grain boundaries extracted from the EBSD image overlaid on the reference BSE image. These points that have a disorientation greater than $5^{\circ}$ with neighbors are considered as grain boundaries. The magnified views (Figures 16(b)] 16(c) and $16(\mathrm{~d})$ ) show that the grain boundaries extracted from EBSD pictures are always several pixels away from the grain boundaries shown in the BSE modality. This is due to the stable distance between the incident beam and the effective back-scattering electron source.

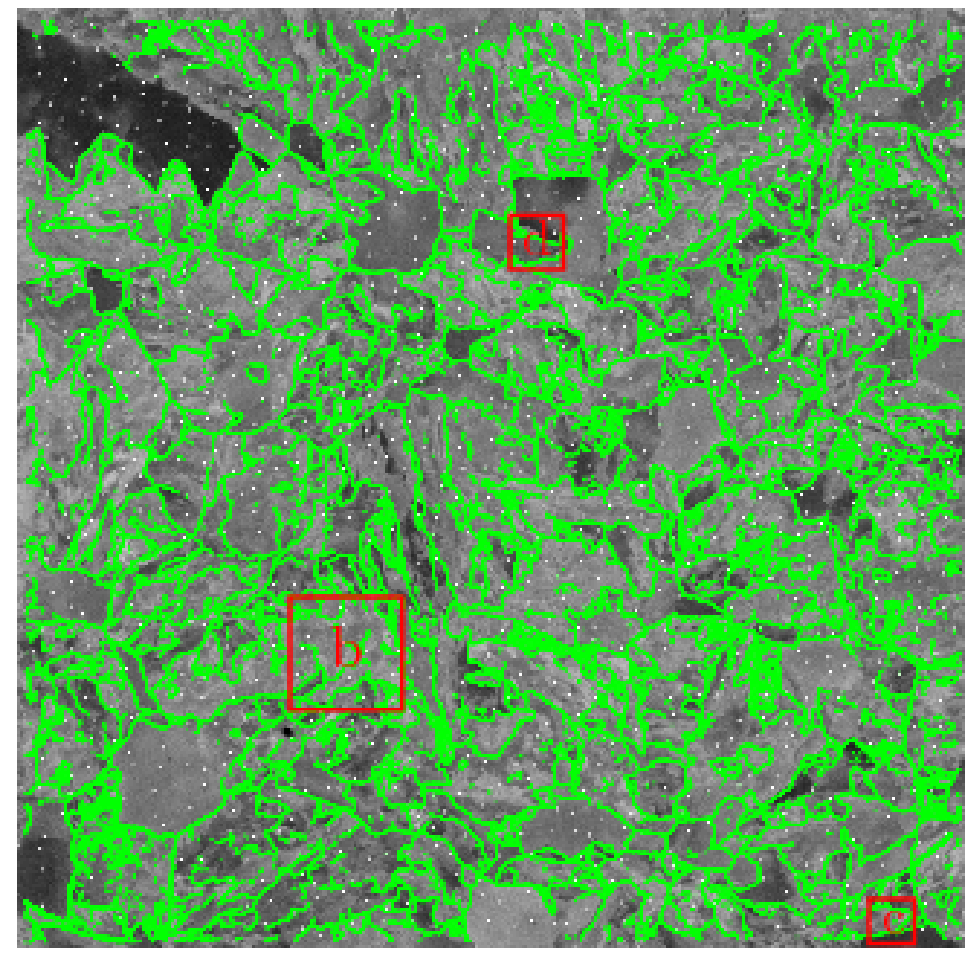

(a)

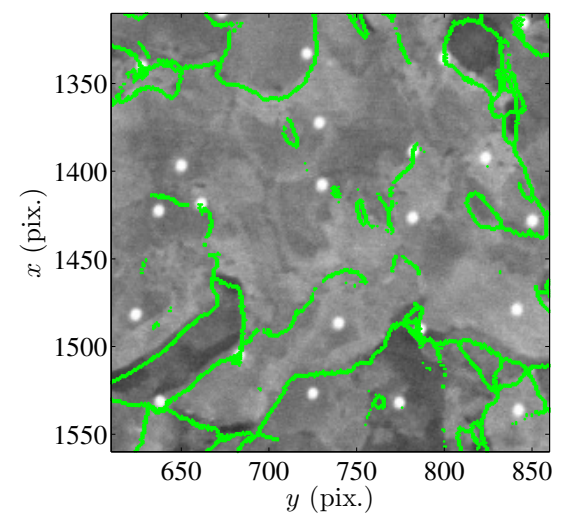

(b)

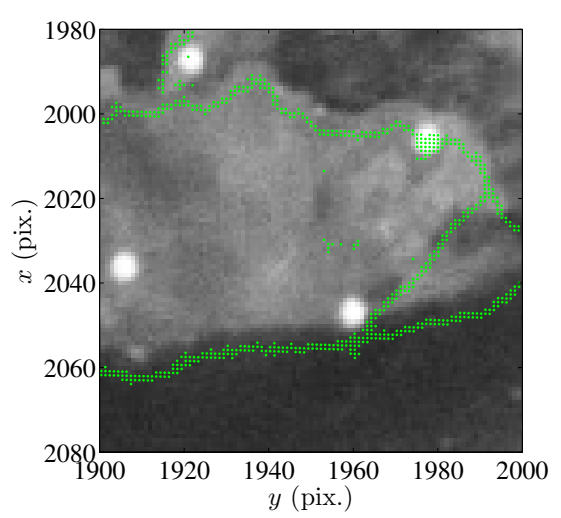

(c)

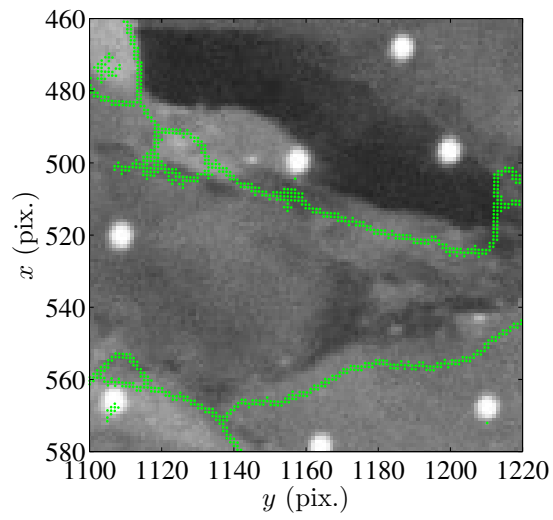

(d)

Figure 16: Overlay of grain boundary extracted from EBSD image on reference BSE image. Full-field (a), and three magnified views (b-d). The pixel size is $42 \mathrm{~nm}$ for all images

An illustration of this phenomenon is provided in Figure 17. The electron beam captures both grain boundary and speckle, yet they are actually separated on the sample. Thus the speckles and grain boundaries shown in the IQ field have a consistent shift. Grain boundaries, which are embedded inside the sample, are registered when encompassed by the interaction zone. As for the Pt speckle, its large atomic number/weight and position at the surface mean that it affects the incident beam directly. As a result, the consistent distance shown in Figure 16 can be used to quantify the distance from the incident beam hitting point and the interaction zone. 


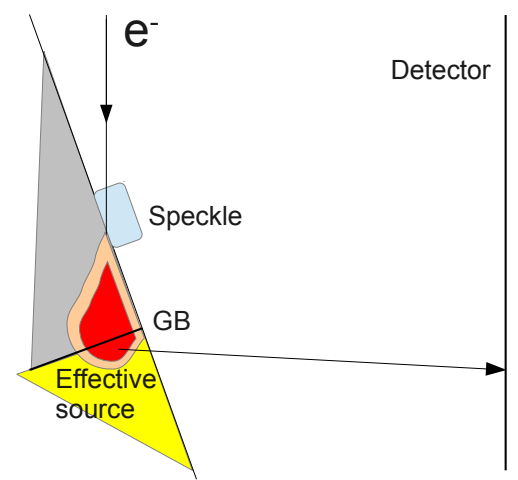

Figure 17: Schematic view of the distance between grain boundaries and real speckle. The electron beam captures both grain boundary and speckle, yet they are actually separated

Figure 18 shows the grain boundary extracted from the EBSD image overlaid on top of the reference BSE image with a constant 7-pixel shift. The magnified views (Figures 18(b), 18(c) and 18(d) show that the accuracy of grain

boundary positioning is about one pixel, which is deemed very satisfactory. 


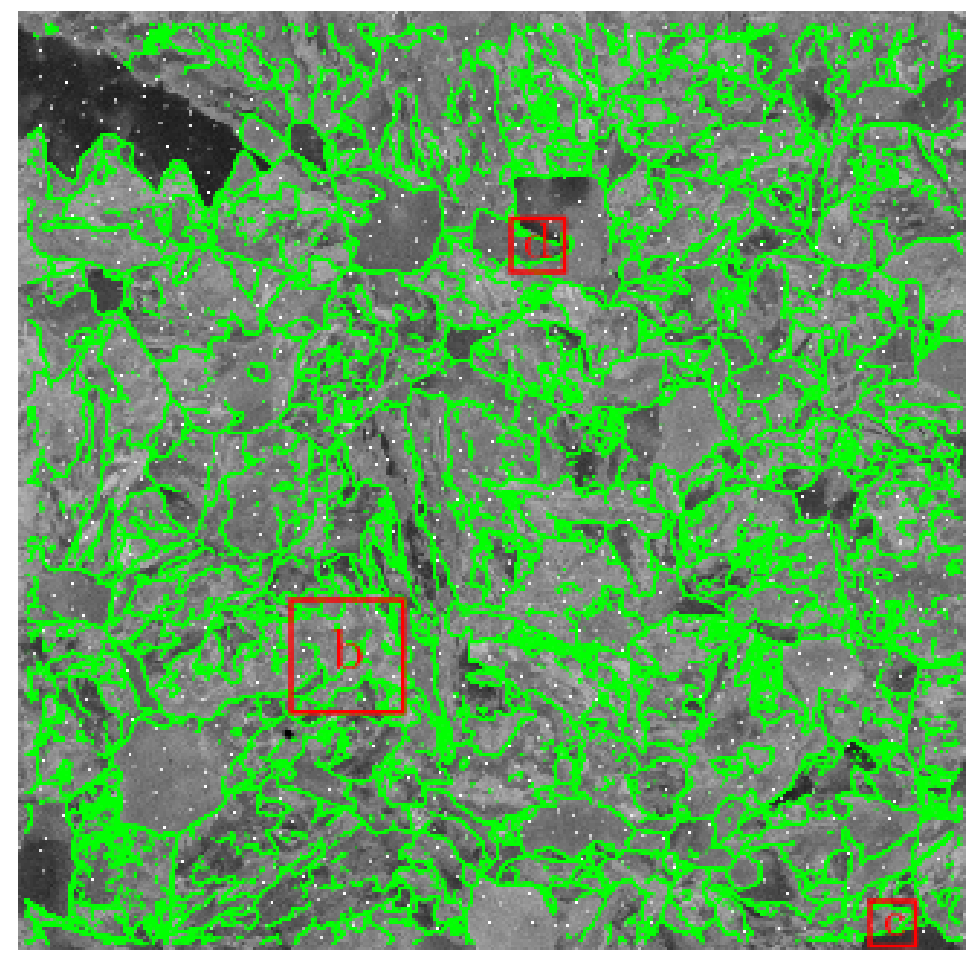

(a)

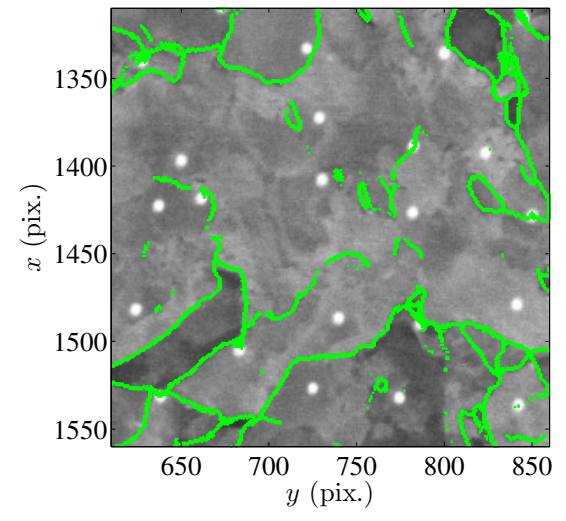

(b)

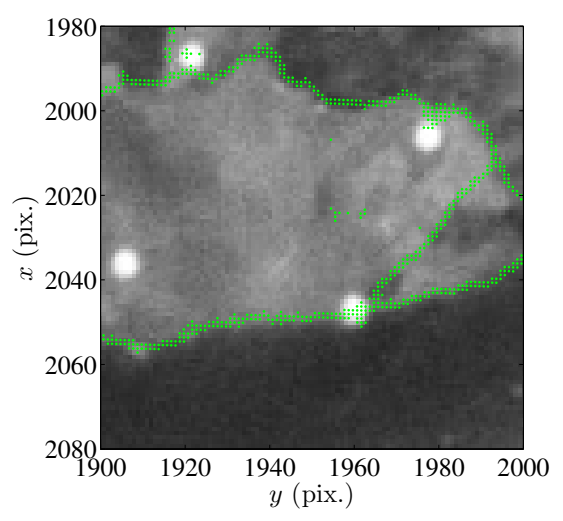

(c)

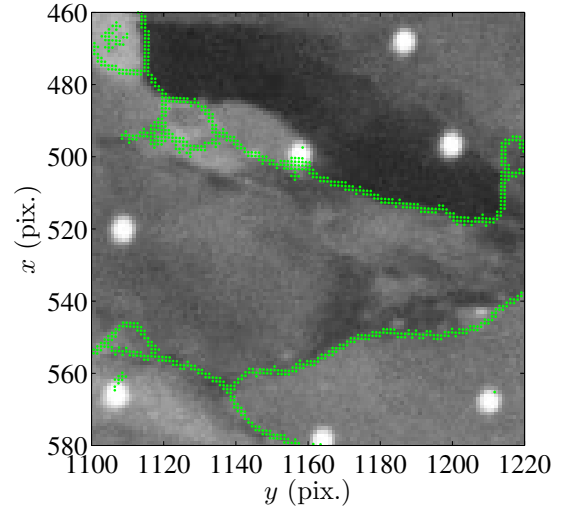

(d)

Figure 18: Overlay of grain boundary extracted from EBSD image on reference BSE image when grain boundaries are uniformly shifted by 7 pixels. Full-field (a), and three magnified views (b-d). The pixel size is $42 \mathrm{~nm}$ for all images

\subsection{Correlation between crystal orientation and plastic strain}

The precise registration between EBSD and BSE images opens another route, which is to study the relationship between crystal rotations and plastic strains in the same coordinate system. An equivalent strain, $\epsilon_{e q}$, is chosen to characterize the strain levels [9]

$$
\epsilon_{e q}=\frac{2}{3}\left[\frac{\sqrt{2 \epsilon_{Y}+1}}{\sqrt{2 \epsilon_{X}+1}}-1\right]
$$

where $\epsilon_{Y}$ and $\epsilon_{X}$ are the principal/eigen values of the in-plane Green-Lagrange strain tensor. The third out-of-plane principal value is assumed to be equal to the second eigen value, $\epsilon_{X} \cdot \epsilon_{e q}$ is a measure of the deviatoric strain.

Figure 19 shows a direct comparison between crystal rotations and equivalent plastic strain for the analyzed pictures. The white pixels in Figure 19(a) come from the imprecise orientation indexation at grain boundaries, and hence quaternion correlation naturally provide a local estimate of the crystal rotation that is meaningless but on average much higher that the trustful crystal rotation within grains. Thus the white pixels draw very precisely 
the grain boundaries [5]. In Figure 19(b) green curves are overlaid grain boundaries thanks to the registration of EBSD and BSE images.

In order to study the correlation between crystal rotations and equivalent plastic strains, a 2D joint histogram is shown in Figure 19(c) for all grains in the region of interest. Figure 19(d) and Figure 19(e) show the 2D joint histogram for ferritic and bainitic grains respectively. Pixels at the grain boundaries are not counted since the measured crystal rotation is not reliable. The three histograms are based on 3.3 million, 0.6 million and 2.7 million data points respectively. By comparing Figure 19(d) and Figure 19(e) it is concluded that strains are generally higher in ferritic grains than in bainitic grains, as found in previous works [39, while no significant differences exist in terms of crystal rotation between the two phases. Strain and rotation are broadly distributed in the three histograms. However, they appear to be significantly correlated, thereby indicating the existence of crystal rotations and plastic strain concentrations in the same regions. To better highlight these correlations, average and median crystal rotations are calculated at fixed $\epsilon_{e q}$ (within intervals of width 0.002), and average and median $\epsilon_{e q}$ are calculated as functions of crystal rotation (within intervals of $0.1^{\circ}$ ). In the statistically significant box, $0.03<\epsilon_{e q}<0.15$ and $3^{\circ}<\theta<10^{\circ}$, all four trends show a marked positive correlation. This analysis does not aim at revealing a deterministic relationship between both of these quantities as they remain very broadly distributed and not only because of measurement uncertainties. A prosaic argument to support this observation is to note that in regions where the strain is small, crystal rotations are expected to remain small.

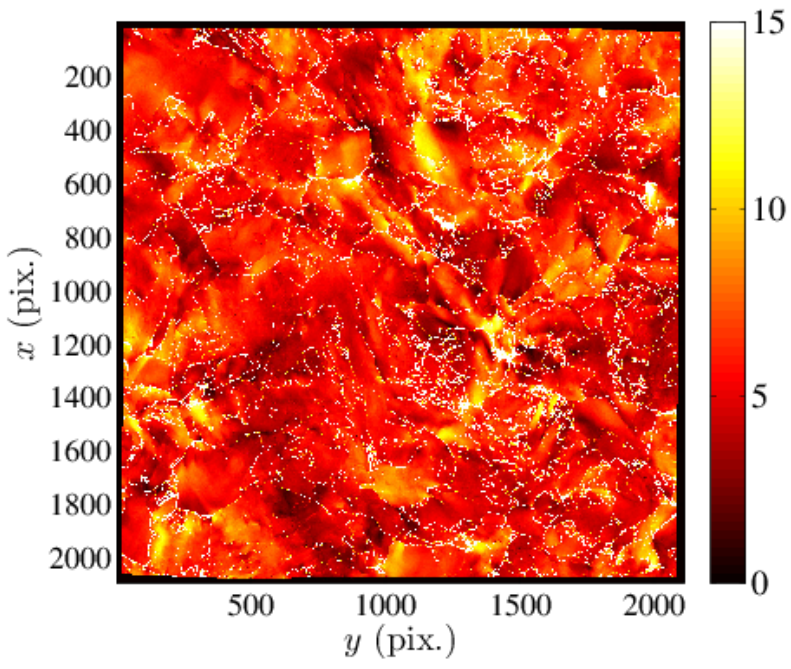

(a)

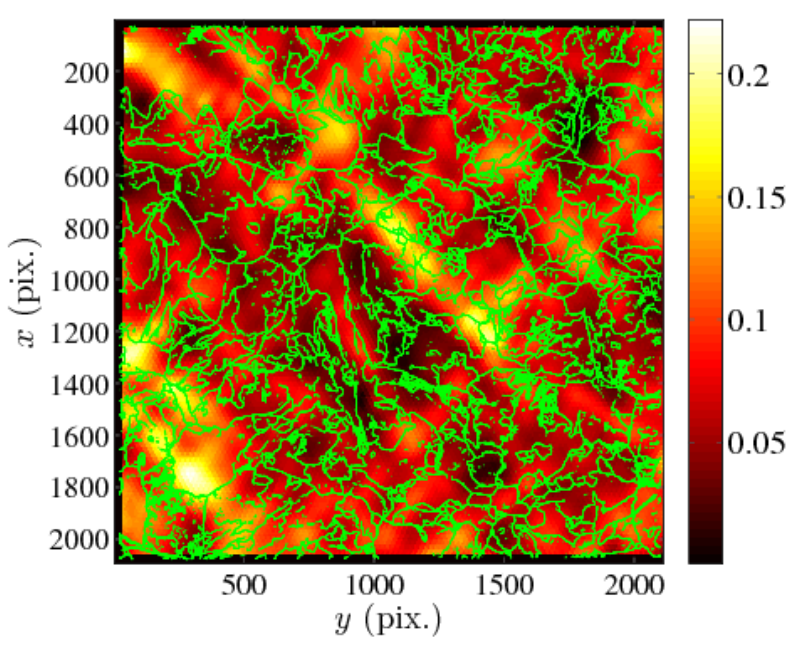

(b) $\epsilon_{e q}$

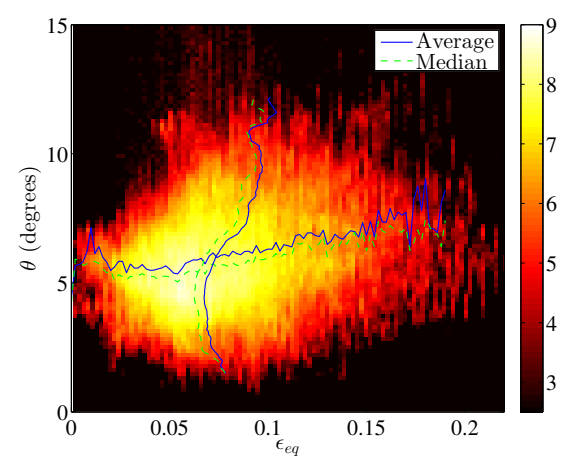

(c) $\log (\rho)$

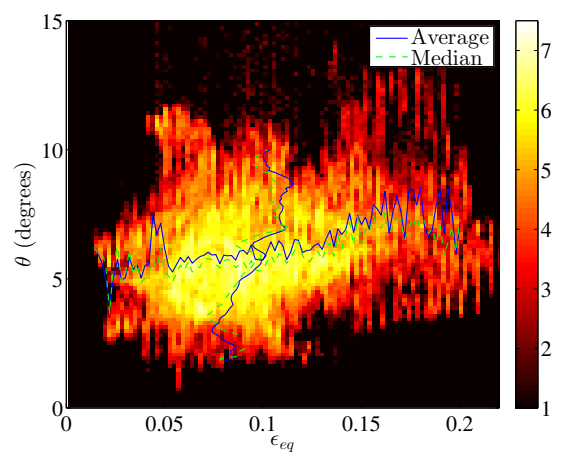

(d) $\log (\rho)$

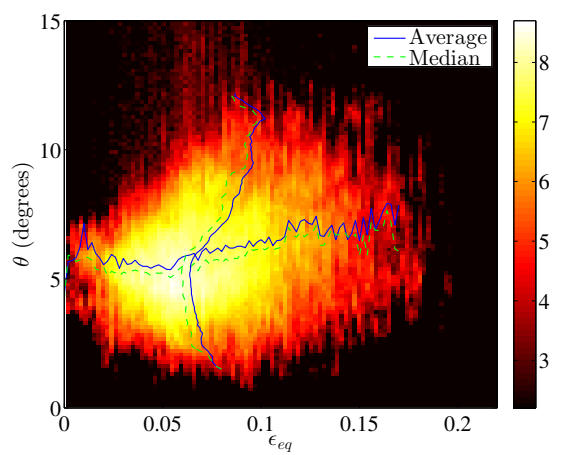

(e) $\log (\rho)$

Figure 19: Comparison of crystal rotations and equivalent plastic strains at the end of the tensile test. (a) Crystal rotation magnitude. (b) Equivalent plastic strain. Grain boundaries extracted from the EBSD image are overlaid. The pixel size is $50 \mathrm{~nm}$ for (a) and (b). 2D joint histograms of crystal rotations and equivalent plastic strains for (c) all grains, (d) ferritic grains and (e) bainitic grains. Note that the scale is in exponential terms. Average and median profiles of crystal rotation and $\epsilon_{e q}$ are overlaid in the sub-figures 


\section{Conclusion}

The present paper provides a detailed study of an in situ tensile test carried out inside an SEM. Properly sized and randomly positioned platinum speckles deposited onto the sample surface facilitate the registration between different modalities including BSE and EBSD images. Regularized DIC reveals very powerful to match BSE images acquired at each increment of mechanical loading, in spite of very heterogeneous (i.e., emerging shear-bands) strain stages. Several new findings have been reported:

1. Properly sized platinum speckles deposited onto the sample surface facilitate the registration of EBSD and BSE/SE images. When finely tuned to the suited value, the speckle thickness may not degrade the reliability of orientation detection of the underlying crystal, and yet leave clear traces in the image quality map. Such a tailoring of the Pt speckle marks reveals very instrumental for further registrations.

2. SEM images suffer from a slow-scan direction error for the SEM device tested herein. Registration of images taken for different scan directions helps to correct this artifact. However, it is worth noting that such artifacts do not impede a quantitative analysis of the surface kinematics with DIC.

3. EBSD acquisitions suffer from unstable electron beam scan, which undermines the precision of coordinates in EBSD results. Yet, DIC based on the Pt speckle seen in BSE and from the IQ map of EBSD enables this artifact to be corrected.

4. Crystallographic orientations indexed by EBSD have an uncertainty of $0.5^{\circ}$. Repeated acquisitions without any change on the sample, reveal from quaternion correlation that crystal orientation and sample position could drift in time with effects as large as $2^{\circ}$ spurious rotation. Quaternion correlation allows such drifts to be corrected, but it shows that an absolute measurement is not accessible.

5. In the EBSD image quality map, each speckle appears as two separate spots. One spot is created when the incident electron beam hitting the Pt disk diminishes the number of electrons interacting with the underlying sample. The other spot is due to back-scattered electrons hitting the Pt speckle, and resulting in a shadow on the Kikuchi diffraction image. Between the two spots a narrow zone may be well indexed, when both above-mentioned phenomena are avoided.

6. The registration of EBSD and BSE images based on speckles exhibits a consistent shift of grain boundaries (evaluated to 7 pixels $/ 350 \mathrm{~nm}$ in the present study). This shift is interpreted as due to the penetration distance of electrons in the EBSD configuration. After the spurious shift is corrected, a pixel-level overlap of EBSD and BSE images is obtained. Thus a very precise description of the polycrystalline microstructure and its changes can be trustfully studied.

7. After registration, full surface plastic strains and crystal rotations are measured and faithfully registered onto the corresponding material points.

The ability to bring in coincidence information coming from the different modalities of SEMs is extremely precious to analyze crystal plasticity from in situ tensile test. Providing proper patterns that are visible in the or chemical etching pits. An interesting scenario is to fill the pits with amorphous Pt to benefit from speckles and yet avoid its shadowing effect. Such suggestions have not been tested.

Exploitation of the present results, which is outside the scope of the present study, is to be performed through a precise registration of these experimental data with a polycrystal model of the very same microstructure that can be simulated using crystal plasticity laws. Invaluable information can be extracted from this synergy between experiments and simulations, for example well designed constitutive equations and their parameters [20, 40, 41, 42, 4, 43, 44.

To mention a final and very ambitious experimental perspective, 3D EBSD from FIB etching of the sample, is a very appealing means of complementing the microstructure and crystallography in the depth. However, being destructive, this technique is only accessible post-mortem, but it may provide one of the last missing pieces of information for reaching an exhaustive characterization of such polycrystalline materials.

\section{Acknowledgments}

The authors acknowledge the financial support of Euratom research and training program 2014-2018 SOTERIA under grant agreement No. 661913. This paper reflects only the authors' view and that the Commission is not responsible for any use that may be made of the information it contains. 
[1] M. Sutton, N. Li, D. Garcia, N. Cornille, J.-J. Orteu, S. McNeill, H. Schreier, X. Li, A. Reynolds, Scanning electron microscopy for quantitative small and large deformation measurements Part II: Experimental validation for magnifications from 200 to 10.000, Experimental Mechanics 47 (6) (2007) 789-804. doi:10.1007/s11340-007-9041-0.

[2] M. Sutton, N. Li, D. Joy, A. Reynolds, X. Li, Scanning electron microscopy for quantitative small and large deformation measurements Part I: SEM imaging at magnifications from 200 to 10.000, Experimental Mechanics 47 (6) (2007) 775-787. doi:10.1007/s11340-007-9042-z.

[3] A. Guery, F. Latourte, F. Hild, S. Roux, Characterization of sem speckle pattern marking and imaging distortion by digital image correlation, Measurement Science and Technology 25 (2014) 12pp. doi: $10.1088 / 0957-0233 / 25 / 1 / 015401$

[4] H. Lim, J. Carroll, C. Battaile, B. Boyce, C. Weinberger, Quantitative comparison between experimental measurements and CP-FEM predictions of plastic deformation in a tantalum oligocrystal, International Journal of Mechanical Sciences 92 (0) (2015) 98 - 108. doi:http://dx.doi.org/10.1016/j.ijmecsci.2014.12.010.

[5] Q. Shi, F. Latourte, F. Hild, S. Roux, Quaternion correlation for tracking crystal motions, Measurement Science and Technology 27 (9) (2016) 095006. doi:https://doi.org/10.1088/0957-0233/27/9/095006.

[6] A. Acharya, J. L. Bassani, A. Beaudoin, Geometrically necessary dislocations, hardening, and a simple gradient theory of crystal plasticity, Scripta Materialia 48 (2) (2003) 167-172. doi:10.1016/S1359-6462(02)00337-8.

[7] S. Wulfinghoff, S. Forest, T. Böhlke, Strain gradient plasticity modeling of the cyclic behavior of laminate 405 microstructures, Journal of the Mechanics and Physics of Solids 79 (2015) 1-20. doi:http://dx.doi.org/ $10.1016 / j \cdot j m p s .2015 .02 .008$.

[8] E. Héripré, M. Dexet, J. Crépin, L. Gélébart, A. Roos, M. Bornert, D. Caldemaison, Coupling between experimental measurements and polycrystal finite element calculations for micromechanical study of metallic materials, International Journal of Plasticity 23 (9) (2007) 1512-1539. doi:10.1016/j.ijplas.2007.01.009.

${ }_{410}^{\text {[ [ }}$ 9] A. Allais, M. Bornert, T. Bretheau, D. Caldemaison, Experimental characterization of the local strain field in an heterogeneous elastoplastic material, Acta Metallurgica Materialia 42 (11) (1994) 3865-3880. doi:http: //dx.doi.org/10.1016/0956-7151(94)90452-9

[10] T. Berfield, J. Patel, R. Shimmin, P. Braun, J. Lambros, N. Sottos, Micro- and nanoscale deformation measurement of surface and internal planes via digital image correlation, Experimental Mechanics 47 (2007) 51-62. doi:10.1007/s11340-006-0531-2.

[11] W. Scrivens, Y. Luo, M. Sutton, S. Collette, M. Myrick, P. Miney, P. Colavita, A. Reynolds, X. Li, Development of patterns for digital image correlation measurements at reduced length scales, Experimental Mechanics 47 (2007) 63-77. doi:10.1007/s11340-006-5869-y.

[12] Z. Xu, X. Li, M. Sutton, N. Li, Drift and spatial distortion elimination in atomic force microscopy images by the digital image correlation technique, Journal of Strain Analysis For Engineering Design 43 (8) (2008) 729-743. doi:10.1243/03093247JSA400.

[13] H. Teyssedre, S. Roux, G. Régnier, A. Tracz, Filtering out slow-scan drifts in atomic force microscopy images, The Journal of Strain Analysis for Engineering Design 46 (5) (2011) 361-367. arXiv:http://dx.doi.org/ 10.1177/0309324711401794, doi:10.1177/0309324711401794

425 [14] J. Venables, R. Bin-jaya, Accurate microcrystallography using electron back-scattering patterns, Philosophical Magazine 35 (5) (1977) 1317-1332. arXiv:http://dx.doi.org/10.1080/14786437708232955, doi:10.1080/ 14786437708232955

[15] F. Ram, S. Zaefferer, T. Jäpel, D. Raabe, Error analysis of the crystal orientations and disorientations obtained by the classical electron backscatter diffraction technique, Journal of Applied Crystallography 48 (3) (2015) 797-813. doi:10.1107/S1600576715005762.

[16] J. Kapur, D. Casasent, Geometric correction of sem images, Proc. SPIE 4044 (2000) 165-176. doi:10.1117/ 12.391928 
[17] C. Wu, B. Adams, C. Bauer, D. Casasent, A. Morawiec, S. Ozdemir, A. Talukder, Mapping the mesoscale interface structure in polycrystalline materials, Ultramicroscopy 93 (2) (2002) 99 - 109. doi:http://dx.doi. org/10.1016/S0304-3991(02)00151-1.

[18] G. Nolze, Geometrically caused image distortion effects and their influence on interpretation of ebsd measurements, Materials Science and Technology 22 (11) (2006) 1343-1351. arXiv:http://dx.doi.org/10.1179/ 174328406X130894, doi:10.1179/174328406X130894.

[19] G. Nolze, Image distortions in SEM and their influences on EBSD measurements, Ultramicroscopy 107 (2-3) (2007) 172 - 183. doi:http://dx.doi.org/10.1016/j.ultramic.2006.07.003.

[20] T. Hoc, J. Crépin, L. Gélébart, A. Zaoui, A procedure for identifying the plastic behavior of single crystals from

n the local response of polycrystals, Acta Materialia 51 (18) (2003) 5477-5488. doi:10.1016/S1359-6454(03) 00413-0.

[21] A. Guery, F. Hild, F. Latourte, S. Roux, Slip activities in polycrystals determined by coupling dic measurements 445 with crystal plasticity calculations, International Journal of Plasticity 81 (2016) 249 - 266. doi:http://dx. doi.org/10.1016/j.ijplas.2016.01.008.

[22] H. Jin, W. Lu, S. Haldar, H. Bruck, Microscale characterization of granular deformation near a crack tip, Journal of Materials Science 46 (20) (2011) 6596-6602. doi:10.1007/s10853-011-5608-3.

[23] F. D. Gioacchino, J. Q. da Fonseca, An experimental study of the polycrystalline plasticity of austenitic 450 stainless steel International Journal of Plasticity 74 (2015) 92-109. doi:http://dx.doi.org/10.1016/j. ijplas.2015.05.012.

[24] M. Sutton, Computer vision-based, noncontacting deformation measurements in mechanics: a generational transformation, Applied Mechanics Reviews 65 (5) (2013) 050802. doi:doi:10.1115/1.4024984.

[25] M. Sutton, W. Wolters, W. Peters, W. Ranson, S. McNeill, Determination of displacements using an improved 455 digital correlation method, Image and Vision Computing 1 (3) (1983) 133 - 139. doi:http://dx.doi.org/ 10.1016/0262-8856(83) 90064-1.

[26] Z. Khan-Jetter, T. Chu, Three-dimensional displacement measurements using digital image correlation and photogrammic analysis, Experimental Mechanics 30 (1) (1990) 10-16. doi:10.1007/BF02322695.

[27] H. Schreier, M. Sutton, J. Orteu, Image Correlation for Shape, Motion and Deformation Measurements: Basic Concepts, Theory and Applications, Springer London, Limited, 2009.

[28] T. Zhu, M. A. Sutton, N. Li, J. J. Orteu, N. Cornille, X. Li, A. P. Reynolds, Quantitative stereovision in a scanning electron microscope, Experimental Mechanics 51 (1) (2011) 97-109. doi:10.1007/s11340-010-9378-7.

[29] B. Bay, T. Smith, D. Fyhrie, M. Saad, Digital volume correlation: three-dimensional strain mapping using X-ray tomography, Exp. Mech. 39 (1999) 217-226. doi:10.1007/BF02323555.

[30] M. Bornert, J. Chaix, P. Doumalin, J. Dupré, T. Fournel, D. Jeulin, E. Maire, M. Moreaud, H. Moulinec, Mesure tridimensionnelle de champs cinématiques par imagerie volumique pour l'analyse des matériaux et des structures, Inst. Mes. Métrol. 4 (2004) 43-88.

[31] E. Verhulp, B. van Rietbergen, R. Huiskes, A three-dimensional digital image correlation technique for strain measurements in microstructures, J. Biomech. 37 (9) (2004) 1313-1320. doi:http://dx.doi.org/10.1016/ j.jbiomech.2003.12.036

[32] S. Roux, F. Hild, P. Viot, D. Bernard, Three-dimensional image correlation from x-ray computed tomography

a of solid foam, Composites Part A: Applied Science and Manufacturing 39 (8) (2008) 1253-1265. doi:10.1016/ j.compositesa.2007.11.011.

[33] F. Hild, S. Roux, Digital Image Correlation, Wiley-VCH, Weinheim (Germany), 2012, pp. 183-228.

475 [34] Z. Tomičević, F. Hild, S. Roux, Mechanics-aided digital image correlation, Journal of Strain Analysis for Engineering Design 48 (2013) 330-343. doi:10.1177/0309324713482457. 
[35] J. Garnaes, L. Nielsen, K. Dirscherl, J. Jørgensen, J. Rasmussen, P. Lindelof, C. Sørensen, Two-dimensional nanometer-scale calibration based on one-dimensional gratings, Applied Physics A 66 (1) (1998) S831-S835. doi:10.1007/s003390051251.

[36] Y. Sun, J. H. Pang, AFM image reconstruction for deformation measurements by digital image correlation, Nanotechnology 17 (4) (2006) 933. doi:https://doi.org/10.1088/0957-4484/17/4/016.

[37] S. Zaefferer, On the formation mechanisms, spatial resolution and intensity of backscatter kikuchi patterns, Ultramicroscopy 107 (2-3) (2007) 254-266. doi:http://dx.doi.org/10.1016/j.ultramic.2006.08.007.

[38] W. Wisniewski, S. Saager, A. Böbenroth, C. Rüssel, Experimental evidence concerning the significant information depth of electron backscatter diffraction (EBSD), Ultramicroscopy 173 (2017) 1 - 9. doi: http: //dx.doi.org/10.1016/j.ultramic.2016.11.004.

[39] F. Latourte, N. Rupin, M. He, A. Parrot, S. Leclercq, Full field measurements used for assessing industrial issues - Two examples, Procedia IUTAM 4 (0) (2012) 92-105. doi:http://dx.doi.org/10.1016/j.piutam. 2012.05.011.

[40] E. Héripré, Méthode de couplage multi-échelles entre simulations numériques polycristallines et mesures de champs pour l'identification des paramètres de lois de comportement et de fissuration des matériaux métalliques. application à l'étude des alliages TiAl, Ph.D. thesis, Ecole Polytechnique (2006).

[41] A. Ma, F. Roters, D. Raabe, On the consideration of interactions between dislocations and grain boundaries in crystal plasticity finite element modeling theory, experiments, and simulations, Acta Materialia 54 (8) (2006) 2181-2194. doi:http://dx.doi.org/10.1016/j.actamat.2006.01.004.

[42] W. Abuzaid, M. Sangid, J. Carroll, H. Sehitoglu, J. Lambros, Slip transfer and plastic strain accumulation across grain boundaries in hastelloy x, Journal of the Mechanics and Physics of Solids 60 (6) (2012) 1201-1220. doi:http://dx.doi.org/10.1016/j.jmps.2012.02.001.

[43] A. Guery, F. Hild, F. Latourte, S. Roux, Identification of crystal plasticity parameters using dic measurements and weighted FEMU, Mechanics of Materials 100 (2016) $55-71$. doi:http://dx.doi.org/10.1016/ j.mechmat.2016.06.007

[44] L. Morsdorf, O. Jeannin, D. Barbier, M. Mitsuhara, D. Raabe, C. Tasan, Multiple mechanisms of lath martensite plasticity, Acta Materialia 121 (2016) 202-214. doi:http://dx.doi.org/10.1016/j.actamat.2016.09. 006. 